\title{
On the genericity of cuspidal automorphic forms of $\mathrm{SO}(2 n+1)$, II
}

\author{
Dihua Jiang and David Soudry
}

\begin{abstract}
This paper is a continuation of our previous work (D. Jiang and D. Soudry, On the genericity of cuspidal automorphic forms on $\mathrm{SO}_{2 n+1}$, J. reine angew. Math., to appear). We extend Moeglin's results (C. Moeglin, J. Lie Theory 7 (1997), 201-229, 231-238) from the even orthogonal groups to old orthogonal groups and complete our proof of the CAP conjecture for irreducible cuspidal automorphic representations of $\mathrm{SO}_{2 n+1}(\mathbb{A})$ with special Bessel models. We also give a characterization of the vanishing of the central value of the standard $L$-function of $\mathrm{SO}_{2 n+1}(\mathbb{A})$ in terms of theta correspondence. As a result, we obtain the weak Langlands functorial transfer from $\mathrm{SO}_{2 n+1}(\mathbb{A})$ to $\mathrm{GL}_{2 n}(\mathbb{A})$ for irreducible cuspidal automorphic representations of $\mathrm{SO}_{2 n+1}(\mathbb{A})$ with special Bessel models.
\end{abstract}

\section{Introduction}

Let $G$ be a reductive algebraic group defined over a number field $k$. Assume that $G$ is $k$-quasisplit. It is known that irreducible cuspidal automorphic representations $\pi$ of $G(\mathbb{A})$, where $\mathbb{A}$ is the ring of adèles of $k$, may not be generic, i.e. may not have a nonzero Whittaker Fourier coefficient. It is interesting to know how close, in terms of near equivalence, the non-generic cuspidal automorphic representations are to irreducible generic cuspidal automorphic representations. Two irreducible automorphic representations $\pi_{1}$ and $\pi_{2}$ are nearly equivalent if, at almost all places $\nu$ of $k$, the local components $\pi_{1, \nu}$ and $\pi_{2, \nu}$ are equivalent, as representations of $G\left(k_{\nu}\right)$. In [JS], we state the CAP conjecture, which says that, for an irreducible cuspidal automorphic representation $\pi$ of $G(\mathbb{A})$, there exists generic cuspidal data $(P, \sigma)$, where $P=M N$ is a parabolic $k$-subgroup of $G$ and $\sigma$ is an irreducible generic cuspidal automorphic representation of the Levi subgroup $M(\mathbb{A})$, such that $\pi$ is nearly equivalent to an irreducible constituent of the induced representation $\operatorname{Ind}_{P(\mathbb{A})}^{G(\mathbb{A})}(\sigma)$ (see [JS, Conjecture 1.1]). Further remarks on this conjecture and its relation to the Arthur conjecture can be found in [JS].

In [JS], we study the CAP conjecture for $G=\mathrm{SO}_{2 n+1}$, the $k$-split odd special orthogonal group. More precisely, we study the CAP conjecture for irreducible cuspidal automorphic representations of $\mathrm{SO}_{2 n+1}(\mathbb{A})$ with a nonzero special Bessel model. From its definition [JS, §2] a special Bessel model is attached to the sub-regular nilpotent orbit of the corresponding Lie algebra, while a Whittaker model is attached to the regular nilpotent orbit. Hence, the family of irreducible cuspidal automorphic representations of $\mathrm{SO}_{2 n+1}(\mathbb{A})$ that have special Bessel models is expected to be very close to irreducible generic cuspidal automorphic representations. Indeed, in Proposition 2.2 and

Received 14 August 2006, accepted in final form 24 November 2006.

2000 Mathematics Subject Classification 22E, 11F.

Keywords: cuspidal automorphic forms, Langlands functoriality.

The research of the first author is partially supported by NSF grant DMS 0400414 and by the distinguished visiting professorship at The Institute of Mathematics, CAS. The research of the second author is supported by a grant from the Israel Science Foundation.

This journal is (C) Foundation Compositio Mathematica 2007. 


\section{JIANG AND D. SOUDRY}

Theorems 4.1 and 4.6 of [JS], we show almost completely that the CAP conjecture holds for irreducible cuspidal automorphic representations of $\mathrm{SO}_{2 n+1}(\mathbb{A})$ that have special Bessel models. What remained to be done was to check the genericity of the cuspidal data involved in the CAP conjecture for this case. In order to determine the genericity of the cuspidal data, we need to know certain basic results about the theta correspondence for the reductive dual pair $\left(\widetilde{\mathrm{Sp}}_{2 m}(\mathbb{A})\right.$, $\left.\mathrm{SO}_{2 n+1}(\mathbb{A})\right)$. Such results for the theta correspondence for the pair $\left(\mathrm{Sp}_{2 m}(\mathbb{A}), \mathrm{SO}_{2 n}(\mathbb{A})\right.$ ) were established by Moeglin in [Moe97a, Moe97b]. One of the main ingredients of the proofs of Moeglin's theorems is the regularized Siegel-Weil formula [KR94] and its variants. Thanks to the work of Ichino [Ioh01] on the regularized Siegel-Weil formula for $\widetilde{\mathrm{Sp}}_{2 m}(\mathbb{A})$, we are able to apply the arguments in Moeglin's proofs to our current case.

In order to make the paper self-contained, as much as possible, we summarize in $\S \S 2.1$ and 2.2 the details of basic facts about both the local and global Rao cocycles and metaplectic groups. In $\S 2.3$, we prove a variant of the regularized Siegel-Weil formula for $\widetilde{\mathrm{Sp}}_{2 n}(\mathbb{A})$ (Corollary 2.2) based on the work of Ichino [Ioh01] and the local theory of the Siegel-Weil formula [Ral84, KR90a, Zhu07], and then extend the theorem of Moeglin on the regularized Siegel-Weil formula for $\mathrm{SO}_{2 n}$ to any number field, by using the regularization in terms of local $p$-adic Hecke elements (Theorem 2.4).

In $\S 3$, we establish certain basic properties of the theta correspondence for the reductive dual pair $\left(\widetilde{\mathrm{Sp}}_{2 m}, \mathrm{SO}_{2 n+1}\right)$, which are analogues of Moeglin's theorems for the reductive dual pair $\left(\mathrm{Sp}_{2 m}, \mathrm{SO}_{2 n}\right)$. Since our proofs are very similar to Moeglin's in [Moe97a, Moe97b], we either indicate briefly, for the sake of completeness, Moeglin's proof for our case or just refer directly to [Moe97a, Moe97b], for the parts which carry over, word for word. To state these theorems, we need further notation.

Let $Z$ be a symplectic vector space of dimension $2 n$ defined over $k$, and let $V$ be a quadratic vector space of odd dimension $m$ defined over $k$. For a positive integer $b$, denote by $Z_{b}$ the direct sum of the space $Z$ and $b$ copies of two-dimensional $k$-symplectic vector spaces. Similarly, denote by $V_{b}$ the direct sum of the space $V$ and $b$ copies of $k$-hyperbolic planes. Denote by $\widetilde{\operatorname{Sp}}(Z)_{\mathbb{A}}\left(\right.$ or $\left.\widetilde{\operatorname{Sp}}\left(Z_{b}\right)_{\mathbb{A}}\right)$ the corresponding adelic metaplectic group attached to $Z$ (or $Z_{b}$, respectively) and, similarly, by $\mathrm{O}(V)_{\mathbb{A}}$ (or $\left.\mathrm{O}\left(V_{b}\right)_{\mathbb{A}}\right)$ the adelic orthogonal group attached to $V$ (or $V_{b}$, respectively). Let $\psi$ be a non-trivial character of $k \backslash \mathbb{A}$. We denote by $\theta_{\psi, Z}^{V}$ the $\psi$-theta correspondence from $\widetilde{\operatorname{Sp}}(Z)_{\mathbb{A}}$ to $\mathrm{O}(V)_{\mathbb{A}}$, and by $\theta_{\psi, V}^{Z}$ the $\psi$-theta correspondence in the other direction.

THEOREM 1.1. Let $\tilde{\pi}$ be an irreducible, genuine, cuspidal, automorphic representation of $\widetilde{\operatorname{Sp}}(Z)_{\mathbb{A}}$. Assume that $\theta_{\psi^{-1}, Z}^{V}(\tilde{\pi})$ is cuspidal.

(1) The following identity holds

$$
\theta_{\psi, V}^{Z}\left(\theta_{\psi^{-1}, Z}^{V}(\tilde{\pi})\right)=\tilde{\pi}
$$

(2) Let $b$ be a positive integer. Then $\theta_{\psi, V}^{Z_{b}}\left(\theta_{\psi^{-1}, Z}^{V}(\tilde{\pi})\right)$ is orthogonal to all cusp forms on $\widetilde{\operatorname{Sp}}\left(Z_{b}\right)_{\mathbb{A}}$.

(3) Let $b$ be a positive integer, and let $Z^{\prime}$ be a symplectic subspace of $Z$, such that $Z=Z_{b}^{\prime}$. Put, for brevity, $Z^{\prime}=Z_{-b}$. Then

$$
\theta_{\psi, V}^{Z-b}\left(\theta_{\psi^{-1}, Z}^{V}(\tilde{\pi})\right)=0 .
$$

TheOREm 1.2. Let $\sigma$ be an irreducible, cuspidal, automorphic representation of $\mathrm{O}(V)_{\mathbb{A}}$. Assume that $\theta_{\psi^{-1}, V}^{Z}(\sigma)$ is cuspidal.

(1) The following identity holds

$$
\theta_{\psi, Z}^{V}\left(\theta_{\psi^{-1}, V}^{Z}(\sigma)\right)=\sigma
$$

(2) Let $b$ be a positive integer. Then $\theta_{\psi, Z}^{V_{b}}\left(\theta_{\psi^{-1}, V}^{Z}(\sigma)\right)$ is orthogonal to all cusp forms on $\mathrm{O}\left(V_{b}\right)_{\mathbb{A}}$. 
ON THE GENERICITY OF CUSPIDAL AUTOMORPHIC FORMS OF $\mathrm{SO}(2 n+1)$, II

(3) Let $b$ be a positive integer, and let $V_{-b}$ be a quadratic subspace of $V$, such that $V=\left(V_{-b}\right)_{b}$. Then

$$
\theta_{\psi, Z}^{V_{-b}}\left(\theta_{\psi^{-1}, V}^{Z}(\sigma)\right)=0 .
$$

The following theorem is deduced from Theorems 1.1 and 1.2, in exactly the same way as in $[$ Moe97a, $\S 3]$.

Theorem 1.3. Let $\tilde{\pi}$ (respectively, $\sigma$ ) be as in Theorem 1.1 (respectively, Theorem 1.2).

(1) The representation $\theta_{\psi^{-1}, Z}^{V}(\tilde{\pi})$ (respectively, $\theta_{\psi^{-1}, V}^{Z}(\sigma)$ ) is irreducible, and for all positive integers $b, \theta_{\psi^{-1}, Z}^{V_{b}}(\tilde{\pi})$ (respectively, $\left.\theta_{\psi^{-1}, V}^{Z_{b}}(\sigma)\right)$ is orthogonal to all cusp forms on $\mathrm{O}\left(V_{b}\right)_{\mathbb{A}}$ (respectively, $\left.\widetilde{\operatorname{Sp}}\left(Z_{b}\right)_{\mathbb{A}}\right)$.

(2) Let $\tilde{\pi}^{\prime}$ (respectively, $\sigma^{\prime}$ ) be an irreducible cuspidal automorphic representation of $\widetilde{\operatorname{Sp}}\left(Z_{b}\right)_{\mathbb{A}}$ (respectively, $\left.\mathrm{O}\left(V_{b}\right)_{\mathbb{A}}\right)$, where $b$ is a non-negative integer; we assume that $\tilde{\pi}^{\prime}$ is genuine. Then $\theta_{\psi^{-1}, Z_{b}}^{V}\left(\tilde{\pi}^{\prime}\right)=\theta_{\psi^{-1}, Z}^{V}(\tilde{\pi})$ (respectively, $\theta_{\psi^{-1}, V_{b}}^{Z}\left(\sigma^{\prime}\right)=\theta_{\psi^{-1}, V}^{Z}(\sigma)$ ), if and only if $b=0$ and $\tilde{\pi}=\tilde{\pi}^{\prime}$ (respectively, $\sigma=\sigma^{\prime}$ ).

(3) Let $\tilde{\pi}, \tilde{\pi}^{\prime}$ (respectively, $\sigma, \sigma^{\prime}$ ) be two isomorphic, irreducible cuspidal automorphic representations of $\widetilde{\operatorname{Sp}}(Z)_{\mathbb{A}}$ (respectively, of $\left.\mathrm{O}(V)_{\mathbb{A}}\right)$. We assume that $\tilde{\pi}$, $\tilde{\pi}^{\prime}$ are genuine. Then

$$
\theta_{\psi^{-1}, Z}^{V}(\tilde{\pi}) \neq 0 \Leftrightarrow \theta_{\psi^{-1}, Z}^{V}\left(\tilde{\pi}^{\prime}\right) \neq 0,
$$

and

$$
\theta_{\psi^{-1}, V}^{Z}(\sigma) \neq 0 \Leftrightarrow \theta_{\psi^{-1}, V}^{Z}\left(\sigma^{\prime}\right) \neq 0 .
$$

In $\S 4$, we discuss several applications of Theorems 1.1, 1.2, and 1.3. First we establish a criterion for the non-vanishing of the central value of the standard $L$-function, $L\left(\sigma, \frac{1}{2}\right)$ attached to an irreducible generic cuspidal automorphic representation $\sigma$ of $\mathrm{SO}_{2 n+1}(\mathbb{A})$ in terms of the $\psi$-theta lift of $\sigma$ to the Witt tower $\widetilde{\operatorname{Sp}}_{2 m}(\mathbb{A})$ (Theorems 4.1 and 4.2). We prove the following.

TheOREm 1.4. Let $\sigma$ be an irreducible cuspidal automorphic representation of $\mathrm{SO}_{2 n+1}(\mathbb{A})$. Assume that $\sigma$ is generic, i.e. has a nonzero Whittaker Fourier coefficient. Then the $\psi$-theta lift of $\sigma$ to $\widetilde{\operatorname{Sp}}_{2 n}(\mathbb{A}), \widetilde{\theta}_{\psi, n}^{n}(\sigma)_{+}$, is non-trivial if and only if $L\left(\sigma, \frac{1}{2}\right) \neq 0$, where $L(\sigma, s)$ is the standard $L$-function attached to $\sigma$.

See $\S 4$ for comments on the relation of this theorem to Furusawa's work [Fur95] and to the work of Howe and Piatetski-Shapiro [HP83].

In the rest of $\S 4$ we complete our proof of the CAP conjecture for irreducible cuspidal automorphic representations of $\mathrm{SO}_{2 n+1}(\mathbb{A})$ with special Bessel models. In fact, we obtain additional information about the generic cuspidal data in terms of the central value of standard $L$-functions. We state this now. Here, for $\lambda \in k^{*}$, which is not a square, $\alpha_{\lambda}$ denotes the global Hilbert symbol $(\cdot, \lambda)$, and $\chi_{\lambda}$ denotes the character of $\mathrm{SO}_{2 n+1}(\mathbb{A})$, which is the composition of the spinor norm with $\alpha_{\lambda}$. For other unexplained notation, see [JS].

TheOREm 1.5. Let $\sigma$ be an irreducible cuspidal automorphic representation of $\mathrm{SO}_{2 n+1}(\mathbb{A})$. Assume that $\sigma$ has a nonzero Bessel model of special type, i.e. of type $\left(D_{\lambda}, 1, \psi_{n, n-1 ; \lambda}\right)$. Then either $\sigma$ is nearly equivalent to an irreducible generic cuspidal automorphic representation, or $\sigma$ is CAP with respect to generic cuspidal data. More precisely, the following hold.

(1) If the special Bessel model is $k$-split, i.e. $\lambda \in k^{\times}$is a square, then $\sigma$ is generic.

(2) Assume that the special Bessel model is not $k$-split. Then the first occurrence, $m_{0, \psi}(\sigma)$, in the $\psi$-theta lifting tower satisfies

$$
n-1 \leqslant m_{0, \psi}(\sigma) \leqslant n .
$$




\section{JIANG AND D. SOUDRY}

(a) Assume that $m_{0, \psi}(\sigma)=n$. Then either $\sigma$ is nearly equivalent to an irreducible generic cuspidal automorphic representation $\sigma^{\prime}$, such that $L\left(\sigma^{\prime} \otimes \chi_{\lambda}, \frac{1}{2}\right) \neq 0$, or $\sigma$ is CAP with respect to the generic cuspidal data of the form

$$
\left(P_{1} ; \alpha_{\lambda}|\cdot|^{\frac{1}{2}} \otimes \sigma_{n-1}\right),
$$

where $P_{1}$ is the standard parabolic subgroup, whose Levi part is isomorphic to $\mathrm{GL}_{1} \times$ $\mathrm{SO}_{2 n-1}$, and $\sigma_{n-1}$ is irreducible generic cuspidal automorphic representation of $\mathrm{SO}_{2 n-1}(\mathbb{A})$, such that $L\left(\sigma_{n-1} \otimes \chi_{\lambda}, \frac{1}{2}\right)=0$.

(b) Assume that $m_{0, \psi}(\sigma)=n-1$. Then $\sigma$ is a CAP representation. It is CAP either with respect to the generic cuspidal data

$$
\left(P_{1} ;|\cdot|^{\frac{1}{2}} \otimes \sigma_{n-1}\right),
$$

such that $L\left(\sigma_{n-1} \otimes \chi_{\lambda}, \frac{1}{2}\right) \neq 0$; or with respect to the generic cuspidal data

$$
\left(P_{1,1} ;|\cdot|^{\frac{1}{2}} \otimes \alpha_{\lambda}|\cdot|^{\frac{1}{2}} \otimes \sigma_{n-2}\right),
$$

where $P_{1,1}$ is the standard parabolic subgroup, whose Levi part is isomorphic to $\mathrm{GL}_{1} \times$ $\mathrm{GL}_{1} \times \mathrm{SO}_{2 n-3}$, and $L\left(\sigma_{n-2} \otimes \chi_{\lambda}, \frac{1}{2}\right)=0$.

It is not hard now to figure out from Theorem 1.5 the possible global Arthur parameters for irreducible automorphic cuspidal representations of $\mathrm{SO}_{2 n+1}(\mathbb{A})$, which have a Bessel model of special type. We will show, in a future work, that the relevant global Arthur packets can be constructed by theta correspondences.

\section{Notation and preliminaries}

\subsection{The metaplectic group over a local field}

Let $F$ be a local field of characteristic 0 and let $W$ be a vector space of dimension $2 f$ over $F$, equipped with a non-degenerate anti-symmetric form $\langle$,$\rangle . Let \operatorname{Sp}(W)$ denote the corresponding symplectic group acting from the right on $W$. For $F \neq \mathbb{C}, \operatorname{Sp}(W)$ has a unique (up to isomorphism) non-trivial twofold cover $\widetilde{\mathrm{Sp}}(W)$ (the metaplectic group). In this paper, we use the realization of $\widetilde{\mathrm{Sp}}(W)$ in terms of a Rao normalized cocycle $c_{W}$ (see [Ran93]), which may be simply called a Rao cocycle. Thus, the metaplectic group $\widetilde{\mathrm{Sp}}(W)$ is realized as

$$
\{(g, \epsilon) \mid g \in \operatorname{Sp}(W), \epsilon= \pm 1\},
$$

with the group law

$$
\left(g_{1}, \epsilon_{1}\right)\left(g_{2}, \epsilon_{2}\right)=\left(g_{1} g_{2}, \epsilon_{1} \epsilon_{2} c_{W}\left(g_{1}, g_{2}\right)\right)
$$

Let us recall briefly some facts about the Rao cocycle $c_{W}$. See [Ran93] for the details. The definition of a Rao (normalized) cocycle depends on a choice of a symplectic basis

$$
B=\left\{\epsilon_{1}, \ldots, \epsilon_{f}, \epsilon_{-f}, \ldots, \epsilon_{-1}\right\}
$$

of $W$, i.e. $W^{+}=\operatorname{Span}_{k}\left\{\epsilon_{1}, \ldots, \epsilon_{f}\right\}$ and $W^{-}=\operatorname{Span}_{k}\left\{\epsilon_{-1}, \ldots, \epsilon_{-f}\right\}$ are totally isotropic subspaces of $W$, and $\left\langle\epsilon_{i}, \epsilon_{-j}\right\rangle=\delta_{i j}$, for $1 \leqslant i, j \leqslant f$. In particular, $W^{+}$and $W^{-}$are transversal Lagrangians of $W$. (So, we should denote the cocycle by $c_{B}$, but this will make future notation quite cumbersome. Later on, we even drop $W$ from $c_{W}$.)

Let $P \subset \mathrm{Sp}(W)$ be the Siegel parabolic subgroup, which preserves $W^{-}$. Let us now list some properties of the Rao cocycle [Ran93]. 
(a) There is a function $x: \operatorname{Sp}(W) \longrightarrow F^{*} /\left(F^{*}\right)^{2}$, satisfying (1)-(5) as follows.

(1) We have $x(p) \equiv \operatorname{det}_{W^{-}}(p) \bmod \left(F^{*}\right)^{2}, p \in P$.

(2) We have $x\left(p_{1} h p_{2}\right)=x\left(p_{1}\right) x(h) x\left(p_{2}\right)$, for $p_{1}, p_{2} \in P$ and $h \in \operatorname{Sp}(W)$.

(3) For $\left(\begin{array}{cc}A & B \\ C & D\end{array}\right) \in \operatorname{Sp}(W)$, according to the polarization $W=W^{+} \oplus W^{-}$, with $\operatorname{det} C \neq 0$,

$$
x\left(\begin{array}{ll}
A & B \\
C & D
\end{array}\right) \equiv \operatorname{det} C \bmod \left(F^{*}\right)^{2} .
$$

(4) Let $(\cdot, \cdot)$ denote the Hilbert symbol of $F$. Then, for $p_{1}, p_{2} \in P, h_{1}, h_{2} \in \operatorname{Sp}(W)$,

$$
\frac{c_{W}\left(p_{1} h_{1}, h_{2} p_{2}\right)}{c_{W}\left(h_{1}, h_{2}\right)}=\frac{\left(x\left(p_{1}\right), x\left(h_{1}\right)\right)\left(x\left(p_{2}\right), x\left(h_{2}\right)\right)}{\left(x\left(p_{1}\right), x\left(p_{2}\right)\right)\left(x\left(p_{1} p_{2}\right), x\left(h_{1} h_{2}\right)\right)}
$$

and

$$
\frac{c_{W}\left(h_{1} p_{1}^{-1}, p_{1} h_{2}\right)}{c_{W}\left(h_{1}, h_{2}\right)}=\left(x\left(p_{1}\right),-x\left(h_{1}\right) x\left(h_{2}\right)\right) .
$$

(5) For each subset $S \subset\{1, \ldots, f\}$, consisting of $j$ elements, let $\tau_{S}$ be the element of $\operatorname{Sp}(W)$, defined by $\epsilon_{i} \tau_{S}=-\epsilon_{-i}$ and $\epsilon_{-i} \tau_{S}=\epsilon_{i}$, for $i \in S$, and, otherwise, $\tau_{S}$ fixes the remaining elements of the basis $B$. Then the double coset $P \tau_{S} P$ depends only on $j=|S|$. Let $\tau_{j}$ be a choice of $\tau_{S}$, for $|S|=j$. Then $\left\{\tau_{j}\right\}_{j=0}^{f}$ is a set of representatives for $P \backslash \operatorname{Sp}(W) / P$. We have

$$
x\left(\tau_{j}\right)=1 .
$$

Note that the Rao function $g \mapsto x(g)$ is unique by [Ran93, Lemma 5.1].

(b) Let $W_{1}, W_{2}$ be two symplectic spaces of dimensions $2 n_{1}, 2 n_{2}$, respectively. Denote the corresponding symplectic forms by $\langle,\rangle_{W_{1}}$ and $\langle,\rangle_{W_{2}}$. Let $W=W_{1} \oplus W_{2}$ be the direct sum of these two spaces, with the symplectic form $\langle\rangle=,\langle,\rangle_{W_{1}} \oplus\langle,\rangle_{W_{2}}$. Let, for $l=1,2, i_{l}: W_{l} \longrightarrow W$ be the embedding of $W_{l}$ in the $l$ th coordinate, and let $j_{l}: \operatorname{Sp}\left(W_{l}\right) \longrightarrow \mathrm{Sp}(W)$ be the corresponding embedding of symplectic groups. Let $j: \operatorname{Sp}\left(W_{1}\right) \times \operatorname{Sp}\left(W_{2}\right) \longrightarrow \operatorname{Sp}(W)$ be the corresponding direct sum embedding, i.e.

$$
j\left(g_{1}, g_{2}\right)=j_{1}\left(g_{1}\right) j_{2}\left(g_{2}\right)=j_{2}\left(g_{2}\right) j_{1}\left(g_{1}\right), \quad \text { for } g_{l} \in \operatorname{Sp}\left(W_{l}\right) .
$$

Choose symplectic bases

$$
B_{l}=\left\{\epsilon_{1}^{(l)}, \ldots, \epsilon_{n_{l}}^{(l)}, \epsilon_{-n_{l}}^{(l)}, \ldots, \epsilon_{-1}^{(l)}\right\}
$$

for $W_{l}, l=1,2$. Let $B=B_{1} \cup B_{2}$, and order $B$ as follows. Take, first, the image, by $i_{1}$, of the first $n_{1}$ elements of $B_{1}$, then the image, by $i_{2}$, of all elements of $B_{2}$, then the image, by $i_{1}$, of the last $n_{1}$ elements of $B_{1}$. This is a symplectic basis of $W$. Let $c_{l}$ be the Rao normalized cocycle on $\operatorname{Sp}\left(W_{l}\right)$, corresponding to $B_{l}(l=1,2)$, and let $c_{W}$ be the Rao normalized cocycle on $\operatorname{Sp}(W)$, corresponding to $B$. Denote by $x_{l}$ the corresponding $x$-functions on $\operatorname{Sp}\left(W_{l}\right)(l=1,2)$. Then [Ran93, Corollary 5.6] states that, for $g_{1}, g_{1}^{\prime} \in \operatorname{Sp}\left(W_{1}\right)$ and $g_{2}, g_{2}^{\prime} \in \operatorname{Sp}\left(W_{2}\right)$,

$$
\frac{c_{W}\left(j\left(g_{1}, g_{2}\right), j\left(g_{1}^{\prime}, g_{2}^{\prime}\right)\right)}{c_{1}\left(g_{1}, g_{1}^{\prime}\right) c_{2}\left(g_{2}, g_{2}^{\prime}\right)}=\left(x_{1}\left(g_{1}\right), x_{2}\left(g_{2}\right)\right)\left(x_{1}\left(g_{1}^{\prime}\right), x_{2}\left(g_{2}^{\prime}\right)\right)\left(x_{1}\left(g_{1} g_{1}^{\prime}\right), x_{2}\left(g_{2} g_{2}^{\prime}\right)\right) \text {. }
$$

In particular, for $g, h \in \operatorname{Sp}\left(W_{l}\right)$, with $l=1,2$

$$
c_{W}\left(j_{l}(g), j_{l}(h)\right)=c_{l}(g, h) .
$$

Thus, the restriction of the Rao normalized cocycle $c_{W}$ to $\operatorname{Sp}\left(W_{l}\right)$ is the Rao normalized cocycle $c_{l}$. Hence, we can lift, for $l=1,2, j_{l}$ to an embedding $\tilde{j}_{l}: \widetilde{\operatorname{Sp}}\left(W_{l}\right) \longrightarrow \widetilde{\operatorname{Sp}}(W)$ by $\tilde{j}_{l}(g, \epsilon)=\left(j_{l}(g), \epsilon\right)$. We have

$$
\tilde{j}_{1}\left(\tilde{g}_{1}\right) \tilde{j}_{2}\left(\tilde{g}_{2}\right)=\tilde{j}_{2}\left(\tilde{g}_{2}\right) \tilde{j}_{1}\left(\tilde{g}_{1}\right),
$$




\section{JIANG AND D. SOUDRY}

and this defines a homomorphism $\tilde{j}: \widetilde{\operatorname{Sp}}\left(W_{1}\right) \times \widetilde{\mathrm{Sp}}\left(W_{2}\right) \longrightarrow \widetilde{\mathrm{Sp}}(W)$, defined by $\tilde{j}\left(\tilde{g}_{1}, \tilde{g}_{2}\right)=$ $\tilde{j}_{1}\left(\tilde{g}_{1}\right) \tilde{j}_{2}\left(\tilde{g}_{2}\right)$. Its kernel is $\left\{\left(I_{W_{1}}, \epsilon\right),\left(I_{W_{2}}, \epsilon\right) \mid \epsilon= \pm 1\right\}$.

(c) Assume that our local field $F$ is non-archimedean of odd residual characteristic. Denote by $\mathcal{O}$ its ring of integers and by $W(\mathcal{O})$ the lattice over $\mathcal{O}$ spanned by the symplectic basis $B$. Let $K \subset \mathrm{Sp}(W)$ be the stabilizer of $W(\mathcal{O})$. This is a maximal compact subgroup (standard with respect to $B)$. It is known that there is an embedding $\varepsilon: K \longrightarrow \widetilde{\mathrm{Sp}}(W)$ of the form $\varepsilon(r)=(r, \epsilon(r))$. See $\left[M V W 87\right.$, p. 43] for a proof. Note that this means that, for $r_{1}, r_{2} \in K$,

$$
c_{W}\left(r_{1}, r_{2}\right)=\frac{\epsilon\left(r_{1} r_{2}\right)}{\epsilon\left(r_{1}\right) \epsilon\left(r_{2}\right)} .
$$

This embedding is unique, if the residual field has at least four elements. It is not hard to see that, for $r \in P(\mathcal{O})$,

$$
\varepsilon(r)=(r, 1) .
$$

(d) We will be interested in dual pairs $\operatorname{Sp}(Z) \times \mathrm{O}(V)$, inside $\mathrm{Sp}(W)$, where $\mathrm{O}(V)$ is the orthogonal group corresponding to a non-degenerate symmetric bilinear form $Q$ on a vector space $V$ of odd dimension $m=2 l+1$, over $F$, and $\operatorname{Sp}(Z)$ is the symplectic group, corresponding to a $2 n$-dimensional vector space over $F$, equipped with a symplectic form $\langle,\rangle_{Z}$. We view $\mathrm{O}(V)$ as acting from the left on $V$ and $\operatorname{Sp}(Z)$ as acting from the right on $Z$. Thus, $W=Z \otimes V$ is of dimension $2 m n$ over $F$ and is equipped with the symplectic form $\langle\rangle=,\langle,\rangle_{Z} \otimes Q$. Let

$$
\alpha: \operatorname{Sp}(Z) \times \mathrm{O}(V) \longrightarrow \mathrm{Sp}(W)
$$

be the natural homomorphism; it is given by $(z \otimes v) \alpha(g, h)=z g \otimes h^{-1} v$. Let $d_{Q}$ and $h_{Q}$ be the discriminant and the Hasse invariant, respectively, of $Q$. Choose an orthogonal basis $\left\{e_{1}, \ldots, e_{m}\right\}$ of $V$, and put $a_{i}=Q\left(e_{i}, e_{i}\right)$, for $1 \leqslant i \leqslant m$. Choose a symplectic basis $B_{Z}=\left\{z_{1}, \ldots, z_{n}, z_{-n}, \ldots, z_{-1}\right\}$, for $Z$. Denote by $B_{Z}^{+}$the first $n$ elements of $B_{Z}$ and by $B_{Z}^{-}$the last $n$ elements. We also denote by $X$ and $Y$ the transversal Lagrangian subspaces of $Z$ spanned by $B_{Z}^{+}$and $B_{Z}^{-}$, respectively. Let $c$ denote the corresponding Rao normalized cocycle on $\operatorname{Sp}(Z)$. Let

$$
B=B_{Z}^{+} \otimes e_{1} \cup \cdots \cup B_{Z}^{+} \otimes e_{m} \cup a_{m}^{-1} B_{Z}^{-} \otimes e_{m} \cup \cdots \cup a_{1}^{-1} B_{Z}^{-} \otimes e_{1} .
$$

This is a symplectic basis for $W$. Let $c_{W}$ denote the corresponding normalized Rao cocycle on $\operatorname{Sp}(W)$. Then, from [Kud96, pp. 20 and 35] one can get the following equivalence of $c$ and the restriction of $c_{W}$ on $\alpha_{1}(\operatorname{Sp}(Z)) \times \alpha_{1}(\operatorname{Sp}(Z))$, where $\alpha_{1}(g)=\alpha\left(g, I_{V}\right)$. (From [Kud96] one sees clearly that the double cover of $\operatorname{Sp}(W)$ does not split over $\operatorname{Sp}(Z)$ exactly due to the fact that $m$ is odd; $\alpha_{1}$ is equivalent to $c^{m}$.) We have, for $g_{1}, g_{2} \in \operatorname{Sp}(Z)$,

$$
c_{W}\left(\alpha_{1}\left(g_{1}\right), \alpha_{1}\left(g_{2}\right)\right)=\frac{e\left(g_{1} g_{2}\right)}{e\left(g_{1}\right) e\left(g_{2}\right)} c\left(g_{1}, g_{2}\right)
$$

where

$$
e(g)=h_{Q}^{u(g)}\left(x(g),(-1)^{\frac{1}{2} m(m-1)} d_{Q}^{u(g)+1}\right)\left(d_{Q},(-1)^{\frac{1}{2} u(g)(u(g)-1)}\right) .
$$

Here $u(g)$ is determined by writing $g$ in the form $p_{1} \tau_{S} p_{2}$, with $|S|=u(g)$, as in property (a) part (5). Note, also, that

$$
x\left(\alpha_{1}(g)\right) \equiv d_{Q}^{u(g)} x(g)^{m} \bmod \left(F^{*}\right)^{2} .
$$

The restriction of $c_{W}$ to $\alpha_{2}(\mathrm{O}(V)) \times \alpha_{2}(\mathrm{O}(V))$, where $\alpha_{2}(h)=\alpha\left(I_{Z}, h\right)$ is very simple (and the following relation is valid also if $m$ were even). For $h_{1}, h_{2} \in \mathrm{O}(V)$,

$$
c_{W}\left(\alpha_{2}\left(h_{1}\right), \alpha_{2}\left(h_{2}\right)\right)=\left(\operatorname{det} h_{1}, \operatorname{det} h_{2}\right)^{n} .
$$

Let $\widetilde{\mathrm{O}}(V)$ be the group of all pairs $\{(h, \epsilon) \mid h \in \mathrm{O}(V), \epsilon= \pm 1\}$, with the group law

$$
\left(h_{1}, \epsilon_{1}\right)\left(h_{2}, \epsilon_{2}\right)=\left(h_{1} h_{2}, \epsilon_{1} \epsilon_{2}\left(\operatorname{det} h_{1}, \operatorname{det} h_{2}\right)\right) \text {. }
$$


ON THE GENERICITY OF CUSPIDAL AUTOMORPHIC FORMS OF $\mathrm{SO}(2 n+1)$, II

Then $\tilde{\alpha}: \widetilde{\operatorname{Sp}}(Z) \times \widetilde{\mathrm{O}}(V) \longrightarrow \widetilde{\mathrm{Sp}}(W)$, given by

$$
\tilde{\alpha}\left(\left(g, \epsilon_{1}\right),\left(h, \epsilon_{2}\right)\right)=\left(\alpha(g, h), \epsilon_{1} \epsilon_{2}^{n} e(g)\left(d_{Q}^{u(g)} x(g), \operatorname{det} h\right)^{n}\right)
$$

is a homomorphism, which lifts $\alpha$. This follows from (2.4), (2.5), (2.7), and the fact that

$$
c_{W}\left(\alpha_{1}(g), \alpha_{2}(h)\right)=c_{W}\left(\alpha_{2}(h), \alpha_{1}(g)\right)=\left(d_{Q}^{u(g)} x(g), \operatorname{det} h\right)^{n},
$$

which follows from (2.1) and (2.6).

We denote $\tilde{\alpha}_{1}(g, \epsilon)=\tilde{\alpha}\left((g, \epsilon),\left(I_{V}, 1\right)\right)$ and $\tilde{\alpha_{2}}(h, \epsilon)=\tilde{\alpha}\left(\left(I_{Z}, 1\right),(h, \epsilon)\right)$. These homomorphisms from $\widetilde{\operatorname{Sp}}(Z)$ and $\widetilde{\mathrm{O}}(V)$ into $\widetilde{\operatorname{Sp}}(W)$ lift $\alpha_{1}$ and $\alpha_{2}$, respectively. For other dual pairs in the symplectic group, see [Kud94].

(e) We keep the notation of property (d). Let $\psi$ be a non-trivial character of $F$. Let $\omega_{\psi}$ be the Weil representation of $\widetilde{\mathrm{Sp}}(W)$, corresponding to $\psi$. We realize it in the Schrödinger model $S(X \otimes V)$. We will identify $X \otimes V \cong V^{n}$, through $z_{1} \otimes v_{1}+\cdots+z_{n} \otimes v_{n} \longmapsto\left(v_{1}, \ldots, v_{n}\right)$. Then we have the following formulae (see [Kud96, p. 37]).

$$
\begin{aligned}
& \omega_{\psi}\left(\tilde{\alpha}_{1}\left(\left(\begin{array}{cc}
a & \\
& a^{*}
\end{array}\right), \epsilon\right)\right) \phi\left(v_{1}, \ldots, v_{n}\right)=\epsilon \gamma\left(\operatorname{det} a, \psi^{\frac{1}{2}}\right)^{-1}\left(\operatorname{det} a,(-1)^{\frac{1}{2} m(m-1)} d_{Q}\right) \\
& \cdot|\operatorname{det} a|^{m / 2} \phi\left(\left(v_{1}, \ldots, v_{n}\right) a\right) \text {; } \\
& \omega_{\psi}\left(\tilde{\alpha}_{1}\left(\left(\begin{array}{cc}
I_{n} & x \\
& I_{n}
\end{array}\right), \epsilon\right)\right) \phi\left(v_{1}, \ldots, v_{n}\right)=\epsilon \psi\left(\frac{1}{2} \operatorname{tr}\left(\operatorname{Gr}\left(v_{1}, \ldots, v_{n}\right) x w_{n}\right)\right) \phi\left(v_{1}, \ldots, v_{n}\right) ; \\
& \omega_{\psi}\left(\tilde{\alpha_{2}}(h, \epsilon)\right) \phi\left(v_{1}, \ldots, v_{n}\right)=\epsilon^{n} \gamma\left(\operatorname{det} h^{-n}, \psi^{\frac{1}{2}}\right)^{-1} \phi\left(h^{-1} v_{1}, \ldots, h^{-1} v_{n}\right) .
\end{aligned}
$$

Here, the elements of $\operatorname{Sp}(Z)$ are written as matrices with respect to the basis $B_{Z}$. The element $w_{n}$ in (2.10) is the $n \times n$ matrix which has 1 in the second main diagonal and zeroes elsewhere. Gr denotes the Gram matrix. Finally, $\gamma\left(t, \psi^{x}\right)$ is the Weil factor, with respect to the character $\psi^{x}$ $(x \neq 0)$. As is traditional, we normalize $\omega_{\psi} \circ \tilde{\alpha}$ so that the action of $\mathrm{O}(V)$ becomes linear. Thus, we define the following representation, which we still denote by $\omega_{\psi}$, of $\widetilde{\operatorname{Sp}}(Z) \times \mathrm{O}(V)$,

$$
\omega_{\psi}(\tilde{g}, h)=\gamma\left(\operatorname{det} h^{-n}, \psi^{\frac{1}{2}}\right) \omega_{\psi}(\tilde{\alpha}(\tilde{g},(h, 1))),
$$

for $\tilde{g} \in \widetilde{\mathrm{Sp}}(Z)$ and $h \in \mathrm{O}(V)$. Note that the first two formulae in (2.10) remain the same, and the last formula in (2.10) becomes

$$
\omega_{\psi}\left(\left(I_{Z}, 1\right), h\right) \phi\left(v_{1}, \ldots, v_{n}\right)=\phi\left(h^{-1} v_{1}, \ldots, h^{-1} v_{n}\right) .
$$

Sometimes, when we want to emphasize the dual pair, we will re-denote $\omega_{\psi}$ in $(2.11)$ by $\omega_{\psi, Z, Q}$ or $\omega_{\psi, Z \otimes V}$.

\subsection{The metaplectic group over an adèle ring}

Let $k$ be a number field. Let $W$ be a vector space, of dimension $2 f$ over $k$, equipped with a symplectic form $\langle\cdot, \cdot\rangle$. Let

$$
B=\left\{\epsilon_{1}, \ldots, \epsilon_{f} ; \epsilon_{-f}, \ldots, \epsilon_{-1}\right\}
$$

be a symplectic basis of $W$, over $k$, as before. For each place $\nu$ of $k$, let $W_{\nu}=k_{\nu} \otimes_{k} W$. We will redenote $k_{\nu} \otimes \epsilon_{j}=k_{\nu} \epsilon_{j}$, for $|j|=1, \ldots, f$. Denote by $\langle\cdot, \cdot\rangle_{\nu}$ the corresponding symplectic form on $W_{\nu}$. Here $B$ serves as a symplectic basis of $W_{\nu}$ as well. Denote by $c_{W_{\nu}}$ the Rao normalized cocycle on $\operatorname{Sp}\left(W_{\nu}\right)$ corresponding to $B$. For each finite odd place $\nu$, we have an embedding $\varepsilon_{\nu}: K_{\nu} \longrightarrow \widetilde{\operatorname{Sp}}\left(W_{\nu}\right)$ as in property (c) of $\S 2.1$. 


\section{JIANG AND D. SOUDRY}

(a) Let $\mathbb{A}$ be the ring of adèles of $k$ and let $\widehat{\operatorname{Sp}}(W)_{\mathbb{A}}$ be the restricted product $\prod_{\nu}^{\prime} \widetilde{\operatorname{Sp}}\left(W_{\nu}\right)$ with respect to $\left\{\varepsilon_{\nu}\left(K_{\nu}\right) \mid \nu\right.$ is finite and odd $\}$. Let

$$
C^{\prime}=\left\{\prod_{\nu}\left(I, \varepsilon_{\nu}\right) \in \widehat{\operatorname{Sp}}(W)_{\mathbb{A}} \mid \prod_{\nu} \varepsilon_{\nu}=1\right\} .
$$

Then

$$
\widetilde{\mathrm{Sp}}(W)_{\mathbb{A}}:=C^{\prime} \backslash \widehat{\mathrm{Sp}}(W)_{\mathbb{A}}
$$

is a twofold central cover of $\operatorname{Sp}(W)_{\mathbb{A}}$. The projection

$$
\operatorname{pr}: \widetilde{\operatorname{Sp}}(W)_{\mathbb{A}} \longrightarrow \operatorname{Sp}(W)_{\mathbb{A}}
$$

satisfies

$$
\operatorname{pr}\left(C^{\prime} \prod_{\nu \in S}\left(g_{\nu}, \epsilon_{\nu}\right) \prod_{\nu \notin S} \varepsilon_{\nu}\left(k_{\nu}\right)\right)=\prod_{\nu \in S} g_{\nu} \prod_{\nu \notin S} k_{\nu},
$$

where $S$ is a finite, large enough set of places. The kernel of the projection is

$$
C_{2}=\left\{C^{\prime} \prod_{\nu}\left(I, \epsilon_{\nu}\right) \in \widetilde{\operatorname{Sp}}(W)_{\mathbb{A}}\right\} \cong\{ \pm 1\}=\mu_{2} .
$$

Note that the natural projection $\widehat{\operatorname{Sp}}(W)_{\mathbb{A}} \longrightarrow \widetilde{\operatorname{Sp}}(W)_{\mathbb{A}}$ is injective on each $\widetilde{\operatorname{Sp}}\left(W_{\nu}\right)$ and gives a commutative diagram

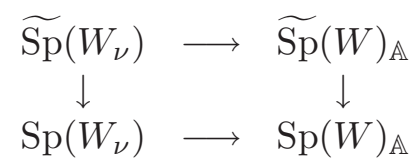

where the vertical arrows are the natural projections.

(b) Let $\gamma \in \operatorname{Sp}(W)$. Let $S$ be a finite set of places, including those at infinity and the even ones, such that $\gamma \in K_{\nu}$, for all $\nu \notin S$. Then there is a finite set of places $S^{\prime} \supset S$, such that

$$
\varepsilon_{\nu}(\gamma)=(\gamma, 1),
$$

for all $\nu \notin S^{\prime}$. This follows from the decomposition $\gamma=p_{1} \tau_{j} p_{2}$ as in property (a) part (5) of $\S 2.1$, where now $p_{1}, p_{2}$ are in $P$, the Siegel $k$-parabolic subgroup of $\operatorname{Sp}(W)$ which stabilizes the subspace $W_{\nu}^{-}=\operatorname{Span}_{k}\left\{\epsilon_{-1}, \ldots, \epsilon_{-f}\right\}$. Thus, we can take $S^{\prime}$, such that $p_{1}, p_{2} \in P\left(k_{\nu}\right) \cap K_{\nu}$, so that $x\left(p_{i}\right) \in \mathcal{O}_{\nu}^{*}$, for all $\nu \notin S^{\prime}(i=1,2)$. By parts (2), (4) and (5) of property (a) in $\S 2.1$, and by (2.3), we get that there is $a_{j}= \pm 1$, such that, for $\nu \notin S^{\prime}$,

$$
\varepsilon_{\nu}(\gamma)=\varepsilon_{\nu}\left(p_{1}\right) \varepsilon_{\nu}\left(\tau_{j}\right) \varepsilon_{\nu}\left(p_{2}\right)=\left(p_{1}, 1\right)\left(\tau_{j}, a_{j}\right)\left(p_{2}, 1\right)=\left(\gamma, a_{j}\right) .
$$

To show that $a_{j}=1$, we write $\tau_{j}$ as a product of elements of the form $\tau_{2}$. By [Ran93, Corollary 5.5], we may assume that $\tau_{j}=\tau_{2}$ and, then, it is a simple calculation in a $\mathrm{SL}_{2}$ to show that $a_{j}=1$.

(c) As a corollary, we get that the map

$$
\gamma \mapsto C^{\prime} \prod_{\nu}(\gamma, 1)
$$

from (the $k$-rational points) $\mathrm{Sp}(W)$ goes to $\widetilde{\mathrm{Sp}}(W)_{\mathbb{A}}$. It is an injective group homomorphism, since for all $\gamma_{1}, \gamma_{2} \in \operatorname{Sp}(W)$, we have

$$
\prod_{\nu} c_{W_{\nu}}\left(\gamma_{1}, \gamma_{2}\right)=1
$$

as follows from Rao's explicit formula in [Ran93, Theorem 5.3]. We view $\operatorname{Sp}(W)$ as a subgroup of $\widetilde{\mathrm{Sp}}(W)_{\mathbb{A}}$, via $(2.15)$. We re-denote the right-hand side of $(2.15)$ by $(\gamma, 1)$. 
(d) Let $P_{\nu}$ be the Siegel parabolic subgroup of $\operatorname{Sp}\left(W_{\nu}\right)$ stabilizing the subspace $W_{\nu}^{-}$as in part (6) of this section and let $P_{\mathbb{A}}$ be the restricted product of all $P_{\nu}$, with respect to $\left\{P_{\nu} \cap K_{\nu} \mid \nu<\infty\right\}$. Denote by $\widetilde{P}_{\mathbb{A}}$, the inverse image of $P_{\mathbb{A}}$ inside $\widetilde{\operatorname{Sp}}(W)_{\mathbb{A}}$. By $(2.13)$, it is easy to see that $\widetilde{P}_{\mathbb{A}} \cong\{(p, \epsilon) \in$ $\left.P_{\mathbb{A}} \times\{ \pm 1\}\right\}$, with the group law

$$
\left(p_{1}, \epsilon_{1}\right)\left(p_{2}, \epsilon_{2}\right)=\left(p_{1} p_{2}, \epsilon_{1} \epsilon_{2} \prod_{\nu}\left(\operatorname{det} p_{1, \nu}, \operatorname{det} p_{2, \nu}\right)_{\nu}\right),
$$

where the determinants of $p_{i, \nu}$ are taken as linear operators on the vector space $W_{\nu}^{-}$. The isomorphism is given by

$$
C^{\prime} \prod_{\nu \in S}\left(p_{\nu}, \epsilon_{\nu}\right) \prod_{\nu \notin S} \varepsilon_{\nu}\left(p_{\nu}\right) \longrightarrow\left(\prod_{\nu} p_{\nu}, \prod_{\nu} \epsilon_{\nu}\right) .
$$

Here $S$ is a large enough finite set, and $p_{\nu} \in P_{\nu} \cap K_{\nu}$, for $\nu \notin S$, so that by $(2.3), \varepsilon_{\nu}\left(p_{\nu}\right)=\left(p_{\nu}, 1\right)$ for $\nu \notin S$. In this case, we also re-denote, for brevity, the elements of $\widetilde{P}_{\mathbb{A}}$ via (2.16) and (2.17) by $(p, \epsilon)$, where $p \in P_{\mathbb{A}}$ and $\epsilon= \pm 1$.

(e) Assume that $W$ is the (orthogonal) direct sum of two symplectic subspaces $W_{1}$ and $W_{2}$. Then, as in property (6) of $\S 2.1$, we have the direct sum embedding $j: \operatorname{Sp}\left(W_{1}\right) \times \operatorname{Sp}\left(W_{2}\right) \longrightarrow \operatorname{Sp}(W)$, and its restriction to each component is given by $j_{l}: \operatorname{Sp}\left(W_{l}\right) \longrightarrow \operatorname{Sp}(W), l=1,2$. Similarly, we have the corresponding local embeddings $j_{\nu}, j_{l, \nu}$, and global embeddings $j_{\mathbb{A}}, j_{l, \mathbb{A}}$. Let us fix symplectic $k$-bases $B_{1}, B_{2}$ and $B=B_{1} \cup B_{2}$ of $W_{1}, W_{2}$, and $W$, respectively, as in property (b) of $\S 2.1$. Then for each place $\nu$, we have obtained the embeddings $\tilde{j}_{l, \nu}: \widetilde{\operatorname{Sp}}\left(W_{l, \nu}\right) \longrightarrow \widetilde{\operatorname{Sp}}\left(W_{\nu}\right), l=1,2$, and the homomorphism $\tilde{j}_{\nu}: \widetilde{\operatorname{Sp}}\left(W_{1, \nu}\right) \times \widetilde{\operatorname{Sp}}\left(W_{2, \nu}\right) \longrightarrow \widetilde{\operatorname{Sp}}\left(W_{\nu}\right)$, which lift $j_{l, \nu}, l=1,2$, and $j_{\nu}$, respectively. Let us denote, for finite and odd places $\nu$, the standard maximal compact subgroup of $\operatorname{Sp}\left(W_{l, \nu}\right)$ by $K_{l, \nu}(l=1,2)$. Consider the embeddings, as in property (c) of $\S 2.1, \varepsilon_{l, \nu}: K_{l, \nu} \longrightarrow \widetilde{\mathrm{Sp}}\left(W_{l, \nu}\right)$. Then, by (2.2) (if the residue field contains at least four elements),

$$
\tilde{j}_{l, \nu}\left(\varepsilon_{l, \nu}(r)\right)=\varepsilon_{\nu}\left(j_{l, \nu}(r)\right)
$$

for $l=1,2$, and $r \in K_{l, \nu}$. Thus, the collection $\left\{\tilde{j}_{l, \nu}\right\}_{\nu}$ defines an embedding $\tilde{j}_{l, \mathbb{A}}: \widetilde{\operatorname{Sp}}\left(W_{l}\right)_{\mathbb{A}} \longrightarrow$ $\widetilde{\operatorname{Sp}}(W)_{\mathbb{A}}$, which lifts $j_{l, \mathbb{A}}$, and a homomorphism $\tilde{j}_{\mathbb{A}}: \widetilde{\operatorname{Sp}}\left(W_{1}\right)_{\mathbb{A}} \times \widetilde{\operatorname{Sp}}\left(W_{2}\right)_{\mathbb{A}} \longrightarrow \widetilde{\operatorname{Sp}}(W)_{\mathbb{A}}$, which lifts $j_{\mathbb{A}}$. We have

$$
\tilde{j}_{\mathbb{A}}\left(\tilde{g}_{1}, \tilde{g}_{2}\right)=\tilde{j}_{1, \mathbb{A}}\left(\tilde{g}_{1}\right) \tilde{j}_{2, \mathbb{A}}\left(\tilde{g}_{2}\right) .
$$

Note that (with our conventions) for $\gamma_{l} \in \operatorname{Sp}\left(W_{l}\right)(l=1,2)$,

$$
\tilde{j}_{\mathbb{A}}\left(\left(\gamma_{1}, 1\right),\left(\gamma_{2}, 1\right)\right)=\left(j\left(\gamma_{1}, \gamma_{2}\right), 1\right) .
$$

(f) Let $\alpha: \operatorname{Sp}(Z) \times \mathrm{O}(V) \longrightarrow \mathrm{Sp}(W)$ be a dual pair, as in property (d) of $\S 2.1$, and we use the same notation, except that here we assume that the vector space $Z$ (respectively $V$ ) and the corresponding non-degenerate anti-symmetric (respectively symmetric) form are defined over the number field $k$. Recall that $\operatorname{dim}_{k} V=m=2 l+1$ is odd. Let $\psi$ be a non-trivial character of $k \backslash \mathbb{A}$. For each local place $\nu$ of $k$, consider the local Weil representation $\omega_{\psi_{\nu}}$ of $\widetilde{\operatorname{Sp}}\left(Z_{\nu}\right) \times \mathrm{O}\left(V_{\nu}\right)$, realized in the Schrödinger model $\mathcal{S}\left(X_{\nu} \otimes V_{\nu}\right) \cong \mathcal{S}\left(V_{\nu}^{n}\right)$. Then the restricted tensor product $\omega_{\psi}=\otimes^{\prime} \omega_{\psi_{\nu}}$ defines the global Weil representation of $\widetilde{\operatorname{Sp}}(Z)_{\mathbb{A}} \times \mathrm{O}(V)_{\mathbb{A}}$ on the Schrödinger model $\mathcal{S}\left(V_{\mathbb{A}}^{n}\right)$. It pulls back to $\widehat{\operatorname{Sp}}(Z)_{\mathbb{A}} \times \mathrm{O}(V)_{\mathbb{A}}$. (Of course, it is obtained from the Weil representation of $\widetilde{\mathrm{Sp}}(Z \otimes V)_{\mathbb{A}}$.) Let $\theta$ be the theta distribution on $S\left(V_{\mathbb{A}}^{n}\right)$,

$$
\theta(\phi)=\sum_{x \in V^{n}} \phi(x)
$$




\section{JIANG AND D. SOUDRY}

and denote, for $(\tilde{g}, h) \in \widetilde{\operatorname{Sp}}(Z)_{\mathbb{A}} \times \mathrm{O}(V)_{\mathbb{A}}$ and $\phi \in S\left(V_{\mathbb{A}}^{n}\right)$,

$$
\theta_{\psi}^{\phi}(\tilde{g}, h)=\theta\left(\omega_{\psi}(\tilde{g}, h) \phi\right)
$$

and sometimes, more precisely,

$$
\theta_{\psi, Z \otimes V}^{\phi}(\tilde{g}, h)=\theta\left(\omega_{\psi, Z \otimes V}(\tilde{g}, h) \phi\right) .
$$

Due to the $\operatorname{Sp}(Z \otimes V)$-invariance of $\theta$, we know that $\theta_{\psi}^{\phi}$ is $\operatorname{Sp}(Z) \times \mathrm{O}(V)$-left invariant. The function $\theta_{\psi}^{\phi}(\tilde{g}, h)$ is of moderate growth in each variable [HP83]. Thus, for an irreducible automorphic representation $\sigma$ of $\mathrm{O}(V)_{\mathbb{A}}$, if either the quadratic form $Q$ on $V$ is anisotropic or $\sigma$ is cuspidal, then the integrals

$$
\theta_{\psi}^{\phi}\left(\varphi_{\sigma}\right)(\tilde{g})=\int_{\mathrm{O}(V) \backslash \mathrm{O}(V)_{\mathbb{A}}} \theta_{\psi}^{\phi}(\tilde{g}, h) \varphi_{\sigma}(h) d h
$$

converge absolutely, for each $\varphi_{\sigma}$, in the space of $\sigma$, and $\phi \in S\left(V_{\mathbb{A}}^{n}\right)$. These automorphic functions $\theta_{\psi}^{\phi}\left(\varphi_{\sigma}\right)$ generate a subspace of automorphic forms on $\widetilde{\operatorname{Sp}}(Z)_{\mathbb{A}}$, which is denoted by $\tilde{\theta}_{\psi, V}^{Z}(\sigma)$. It is stable under right translations by $\widetilde{\operatorname{Sp}}(Z)_{\mathbb{A}}$. We use the same notation, for the automorphic representation of $\widetilde{\operatorname{Sp}}(Z)_{\mathbb{A}}$, thus obtained. This is the automorphic representation of $\widetilde{\operatorname{Sp}}(Z)_{\mathbb{A}}$ obtained by the $\psi$-theta correspondence from $\sigma$, which may of course be zero. Similarly, we consider the $\psi$-theta correspondence in the reverse direction, starting with an irreducible genuine cuspidal automorphic representation $\tilde{\pi}$ of $\widetilde{\operatorname{Sp}}(Z)_{\mathbb{A}}$. The subspace of automorphic functions on $\mathrm{O}(V)_{\mathbb{A}}$ thus obtained is denoted by $\theta_{\psi, Z}^{V}(\tilde{\pi})$. It is an automorphic representation of $\mathrm{O}(V)_{\mathbb{A}}$ in the space generated by the integrals

$$
\theta_{\psi}^{\phi}\left(\varphi_{\tilde{\pi}}\right)(h)=\int_{C_{2} \operatorname{Sp}(Z) \backslash \widetilde{\operatorname{Sp}}(Z)_{\AA}} \theta_{\psi}^{\phi}(\tilde{g}, h) \varphi_{\tilde{\pi}}(\tilde{g}) d \tilde{g},
$$

as $\phi$ and $\varphi_{\tilde{\pi}}$ vary in $S\left(V_{\mathbb{A}}^{n}\right)$ and the space of $\tilde{\pi}$, respectively.

\subsection{The Siegel-Weil formula}

We keep the notation of $\S 2.2$. We establish some simple variants of the regularized Siegel-Weil formulae based on the works of Kudla and Rallis [KR94], Ichino [Ioh01], and Moeglin [Moe97a, Moe97b].

Let $(V, Q)$ be the nondegenerate quadratic vector space over $k$ with Witt index $r$. If $r=0$, the $\psi$-theta lifting of the trivial representation of $\mathrm{O}(V)_{\mathbb{A}}$ to $\widetilde{\operatorname{Sp}}(Z)_{\mathbb{A}}$ does not need regularization. In the following we may assume that $r>0$. We first recall Ichino's formulation [Ioh01] of the Siegel-Weil formula. Assume that $\operatorname{dim}_{k} V=m$ is odd, and that

$$
m=\operatorname{dim}_{k} V<\frac{1}{2} \operatorname{dim}_{k} Z+1=n+1 .
$$

Since $V$ has Witt index $r>0$, we may write $V=V_{0} \oplus H_{r}$, where $V_{0}$ is $k$-anisotropic and $H_{r}$ is the direct sum of $r$ hyperbolic planes. Let $\left(V^{\prime}, Q^{\prime}\right)$ be the nondegenerate quadratic space over $k$ in the same Witt class of $(V, Q)$, and of dimension $m^{\prime}=2 n+2-m$. In this case we may write

$$
V^{\prime}=V_{0} \oplus H_{n+1-r-\operatorname{dim}_{k} V_{0}} .
$$

From (2.20), we have

$$
n+1-r-\operatorname{dim}_{k} V_{0}>m-r-\operatorname{dim}_{k} V_{0}=r>0 .
$$

Ichino's theorem now applies with our $m^{\prime}$ replacing $m$ in [Ioh01, p. 203]. Indeed, let $r^{\prime}=n+1-r-$ $\operatorname{dim}_{k} V_{0}$ denote the Witt index of $V^{\prime}$. Then we clearly have $n+1<m^{\prime} \leqslant 2 n+2$, and $m^{\prime}-r^{\prime} \leqslant n+1$, which are two of the conditions in Ichino's theorem (the regularized Siegel-Weil formula). 
We recall some details of the regularized Siegel-Weil formula from [Ioh01]. In the case under consideration, the theta integral

$$
\int_{\mathrm{O}(V) \backslash \mathrm{O}(V)_{\mathbb{A}}} \theta_{\psi}^{\phi}(\tilde{g}, h) d h
$$

diverges in general. In [KR94], the regularization is carried out using a certain differential operator from the universal enveloping algebra at real archimedean local places. In [Ioh01], the regularization is obtained by using a certain element of a local $p$-adic Hecke algebra. The advantage of the approach in [Ioh01], whose possibility was mentioned in [KR94], is that it applies to any number field.

Let us describe Ichino's regularization. Fix a finite place $\nu_{0}$ of $k$, where the number of elements of the residue field is congruent to 1 modulo 4 . Assume also that $\psi_{\nu_{0}}$ is unramified, and the quadratic form is unimodular at $\nu_{0}$. Assume that $\phi \in \mathcal{S}\left(V_{\mathbb{A}}^{n}\right)$ is fixed by $\omega_{\psi_{\nu_{0}}}\left(\varepsilon_{\nu_{0}}\left(K_{\nu_{0}}^{Z} \times K_{\nu_{0}}^{\prime}\right)\right)$. Here, $K_{\nu_{0}}^{Z}$ is a (standard) maximal compact subgroup of $\operatorname{Sp}\left(Z_{\nu_{0}}\right)$, and $K_{\nu_{0}}^{\prime}$ is a hyper-special maximal compact subgroup of $\mathrm{O}\left(V_{\nu_{0}}\right)$. Then there is an element $\alpha_{\nu_{0}}$, which depends only on the place $\nu_{0}$, in the spherical Hecke algebra (genuine functions) of $\widetilde{\operatorname{Sp}}\left(Z_{\nu_{0}}\right)$ with respect to $K_{\nu_{0}}^{Z}$, such that $\theta_{\psi}^{\omega_{\psi_{0}}\left(\alpha_{\nu_{0}}, 1\right) \phi}(\tilde{g}, h)$ is rapidly decreasing in $h$, and hence the integral

$$
\int_{\mathrm{O}(V) \backslash \mathrm{O}(V)_{\mathbb{A}}} \theta_{\psi}^{\omega_{\psi_{\nu_{0}}}\left(\alpha_{\nu_{0}}, 1\right) \phi}(\tilde{g}, h) d h
$$

converges absolutely. There is a non-zero constant $c_{\nu_{0}}$, such that if the integral $(2.21)$ converges absolutely, then

$$
\int_{\mathrm{O}(V) \backslash \mathrm{O}(V)_{\mathbb{A}}} \theta_{\psi}^{\phi}(\tilde{g}, h) d h=c_{\nu_{0}}^{-1} \int_{\mathrm{O}(V) \backslash \mathrm{O}(V)_{\mathbb{A}}} \theta_{\psi}^{\omega_{\psi_{\nu_{0}}}\left(\alpha_{\nu_{0}}, 1\right) \phi}(\tilde{g}, h) d h .
$$

In other words, the regularization (2.23) is a natural extension of (2.21), in its domain of convergence. Moreover, there is an element $\alpha_{\nu_{0}}^{\prime}=\theta\left(\alpha_{\nu_{0}}\right)$ in the spherical Hecke algebra of $\mathrm{O}\left(V_{\nu_{0}}\right)$, with respect to $K_{\nu_{0}}^{\prime}$, such that

$$
\omega_{\psi_{\nu_{0}}}\left(\alpha_{\nu_{0}}, 1\right)=\omega_{\psi_{\nu_{0}}}\left(1, \alpha_{\nu_{0}}^{\prime}\right)
$$

as endomorphisms of $\mathcal{S}\left(V_{\mathbb{A}}^{n}\right)^{\omega_{\nu_{0}}}\left(\varepsilon_{\nu_{0}}\left(K_{\nu_{0}}^{Z}\right) \times K_{\nu_{0}}^{\prime}\right)$. Here $\theta$ is the homomorphism from the Hecke algebra of $\widetilde{\operatorname{Sp}}\left(Z_{\nu_{0}}\right)$ to the Hecke algebra of $\mathrm{O}\left(V_{\nu_{0}}\right)$, given in [Ioh01, Proposition 1.1]. For $\phi \in \mathcal{S}\left(V_{\mathbb{A}}^{n}\right)$ and $\tilde{g} \in \widetilde{\operatorname{Sp}}(Z)_{\mathbb{A}}$, the regularized theta integral is denoted by

$$
I_{\mathrm{reg}, V, \psi}(\tilde{g}, \phi)=c_{\nu_{0}}^{-1} \int_{\mathrm{O}(V) \backslash \mathrm{O}(V)_{\mathbb{A}}} \theta_{\psi}^{\omega_{\psi_{\nu_{0}}}\left(\alpha_{\nu_{0}}, 1\right) \phi}(\tilde{g}, h) d h .
$$

It is independent of the choice of $\nu_{0}$, as above, or the choice of $\alpha_{\nu_{0}}$. Finally, for $\phi^{\prime} \in \mathcal{S}\left(\left(V_{\mathbb{A}}^{\prime}\right)^{n}\right), s \in \mathbb{C}$, and $\tilde{g} \in \widetilde{\operatorname{Sp}}(Z)_{\mathbb{A}}$, the Siegel section attached to $\phi^{\prime}$ is denoted by

$$
f_{s}^{\phi^{\prime}}(\tilde{g})=|a(\tilde{g})|^{s-(n+1-m) / 2} \omega_{\psi, Z}^{V^{\prime}}\left(\tilde{g}, I_{V^{\prime}}\right) \phi^{\prime}(0) .
$$

Here $a(\tilde{g})$ is defined as follows: if $g \in \operatorname{Sp}(Z)_{\mathbb{A}}$ is the projection of $\tilde{g}$ and $g=p k$ is its Iwasawa decomposition with $p \in P_{\mathbb{A}}$ and $k \in K_{\mathbb{A}}^{Z}$ (with self-evident notation), then $a(\tilde{g})=a(g)=\prod_{\nu} \operatorname{det}_{Y_{\nu}} p_{\nu}$. By comparing with [Ioh01, p. 202] we note that

$$
\frac{m^{\prime}-n-1}{2}=\frac{n+1-m}{2} .
$$

By (2.10) and using part (d) of $\S 2.2$, we have

$$
f_{s}^{\phi^{\prime}}\left(\left(\left(\begin{array}{cc}
a & x \\
& a^{*}
\end{array}\right), \epsilon\right) \tilde{g}\right)=\epsilon \chi_{V^{\prime}, \psi}(\operatorname{det} a)|\operatorname{det} a|^{s+(n+1) / 2} f_{s}^{\phi^{\prime}}(\tilde{g}),
$$




\section{JIANG AND D. Soudry}

where

$$
\chi_{V^{\prime}, \psi}(x)=\prod_{\nu} \chi_{V_{\nu}^{\prime}, \psi_{\nu}}\left(x_{\nu}\right)=\prod_{\nu} \gamma\left(x_{\nu}, \psi_{\nu}^{\frac{1}{2}}\right)^{-1}\left(x_{\nu},(-1)^{\frac{1}{2} m^{\prime}\left(m^{\prime}-1\right)} d_{Q^{\prime}}\right)_{\nu}
$$

Note that

$$
\chi_{V, \psi}=\chi_{V^{\prime}, \psi} \text {. }
$$

Indeed, $(-1)^{\frac{1}{2} m^{\prime}\left(m^{\prime}-1\right)} d_{Q^{\prime}}=(-1)^{\frac{1}{2} m(m-1)} d_{Q}$. We view $\chi_{V, \psi}$ as a character of $\tilde{P}_{\mathbb{A}}$. From $(2.27)$, we see that

$$
f_{s}^{\phi^{\prime}} \in \operatorname{Ind}_{\tilde{P}_{\mathbb{A}}}^{\widetilde{\operatorname{Sp}}(Z)_{\mathbb{A}}}\left(\chi_{V, \psi}|\operatorname{det}|^{s}\right) .
$$

Consider the Eisenstein series, which is the analytic continuation of the following absolutely convergent series when $\operatorname{Re}(s)>n+1 / 2$,

$$
E\left(\tilde{g}, f_{s}^{\phi^{\prime}}\right)=\sum_{\gamma \in P \backslash \operatorname{Sp}(Z)} f_{s}^{\phi^{\prime}}((\gamma, 1) \tilde{g}) .
$$

It has at most a simple pole at $s=(n+1-m) / 2$. Finally, Ichino defines a map $r_{V^{\prime}, V}: \mathcal{S}\left(V_{\mathbb{A}}^{\prime}\right)^{n} \longrightarrow$ $\mathcal{S}\left(V_{\mathbb{A}}^{n}\right)$, which is $\widetilde{\operatorname{Sp}}(Z)_{\mathbb{A}}$-intertwining (this is ' $\pi_{Q^{\prime}}^{Q} \pi_{K}$ ' in [Ioh01]). Now, we can state the following.

Theorem 2.1 (Ichino [Ioh01]). In the notation above, assume that $m<n+1$ (and $m$ is odd). Then there is a non-zero constant $c_{0}$, such that, for all $\phi^{\prime} \in \mathcal{S}\left(\left(V_{\mathbb{A}}^{\prime}\right)^{n}\right)$ and $\tilde{g} \in \widetilde{\operatorname{Sp}}(Z)_{\mathbb{A}}$,

$$
\operatorname{Res}_{s=(n+1-m) / 2} E\left(\tilde{g}, f_{s}^{\phi^{\prime}}\right)=c_{0} I_{\mathrm{reg}, V, \psi}\left(\tilde{g}, r_{V^{\prime}, V}\left(\phi^{\prime}\right)\right) .
$$

We derive the following corollary, which will be used in the following sections.

Corollary 2.2. Assume that $m<n+1$ ( $m$ odd). Then, for all $\phi \in \mathcal{S}\left(V_{\mathbb{A}}^{n}\right)$, there is $\phi^{\prime} \in \mathcal{S}\left(\left(V_{\mathbb{A}}^{\prime}\right)^{n}\right)$, such that for all $\tilde{g} \in \widetilde{\operatorname{Sp}}(Z)_{\mathbb{A}}$,

$$
I_{\mathrm{reg}, V, \psi}(\tilde{g}, \phi)=\operatorname{Res}_{s=(n+1-m) / 2} E\left(\tilde{g}, f_{s}^{\phi^{\prime}}\right) .
$$

Proof. By checking the definition of $r_{V^{\prime}, V}$ (which is ' $\pi_{Q^{\prime}}^{Q} \pi_{K}$ ' in [Ioh01, p. 203]), it is easy to see that at almost all finite places $\nu$, the local map $r_{V_{\nu}^{\prime}, V_{\nu}}$ takes the characteristic function of the standard lattice in $\left(V_{\nu}^{\prime}\right)^{n}$ to the characteristic function of the standard lattice of $V_{\nu}^{n}$. Let $\nu$ be an arbitrary place of $k$, and $\phi=\bigotimes_{\nu^{\prime}} \phi_{\nu^{\prime}}$ be a factorizable function in $\mathcal{S}\left(V_{\AA}^{n}\right)$, whose local components $\phi_{\nu^{\prime}}$ are fixed at all places, except at the given local place $\nu$, where we let it vary. Consider the linear functional

$$
l_{\nu}\left(\phi_{\nu}\right)=I_{\mathrm{reg}, V, \psi}(\tilde{1}, \phi) .
$$

Here, $\tilde{1}$ denotes the identity element of $\widetilde{\operatorname{Sp}}(Z)_{\mathbb{A}}$. We recall from $(2.24)$ in the case $\nu_{0}=\nu$ that

$$
l_{\nu}\left(\omega_{\psi_{\nu}, Z_{\nu}, Q_{\nu}}\left(\tilde{g_{\nu}}, h_{\nu}\right) \phi_{\nu}\right)=I_{\mathrm{reg}, V, \psi}\left(\tilde{g_{\nu}}, \phi\right) .
$$

In particular, $l_{\nu}$ is $\mathrm{O}\left(V_{\nu}\right)$-invariant, i.e. it factors through the space of co-invariants $S\left(V_{\nu}^{n}\right)_{\mathrm{O}\left(V_{\nu}\right)}$. This last space is isomorphic to $\mathrm{R}_{n}\left(V_{\nu}\right)$, which is the space of functions on $\widetilde{\operatorname{Sp}}\left(Z_{\nu}\right)$ generated by $f^{\phi_{\nu}}(\tilde{g})=\omega_{\psi_{\nu}}(\tilde{g}, 1) \phi_{\nu}(0)$, as $\phi_{\nu}$ varies in $\mathcal{S}\left(V_{\nu}^{n}\right)$. Note that

$$
\mathrm{R}_{n}\left(V_{\nu}\right) \subset \operatorname{Ind}_{\tilde{P}_{\nu}}^{\widetilde{\mathrm{Sp}}\left(Z_{\nu}\right)}\left(\chi_{V_{\nu}, \psi_{\nu}}|\operatorname{det}|^{(m-n-1) / 2}\right) .
$$

The isomorphism

$$
d_{\nu}^{\prime}: \mathcal{S}\left(V_{\nu}^{n}\right)_{\mathrm{O}\left(V_{\nu}\right)} \rightarrow \mathrm{R}_{n}\left(V_{\nu}\right)
$$

is induced from $d_{\nu}\left(\phi_{\nu}\right)=f^{\phi_{\nu}}$. See [Ral84] for $\nu$ finite, [KR90a] for $\nu$ real, and [Zhu07] for $\nu$ complex. Thus, if $f^{\phi_{\nu}}=0$, then $l_{\nu}\left(\omega_{\psi_{\nu}}(\tilde{g}, 1) \phi_{\nu}\right)=0$ for all $\tilde{g} \in \widetilde{\operatorname{Sp}}\left(Z_{\nu}\right)$. Consider the map

$$
d_{\nu} \circ r_{V_{\nu}^{\prime}, V_{\nu}}: \mathcal{S}\left(V_{\nu}^{\prime n}\right) \rightarrow \mathrm{R}_{n}\left(V_{\nu}\right)
$$


ON THE GENERICITY OF CUSPIDAL AUTOMORPhic FORMS OF $\mathrm{SO}(2 n+1)$, II

This map is $\widetilde{\operatorname{Sp}}\left(Z_{\nu}\right)$-intertwining and $\mathrm{O}\left(V_{\nu}\right)$-invariant [Ioh01, Lemma 5.5]. By [Ioh01, Lemma 5.1] for $\nu$ finite, [KR90a, Corollary 2.7] for $\nu$ real, and [Zhu07] for $\nu$ complex, and by our assumption that $m<n+1, \mathrm{R}_{n}\left(V_{\nu}\right)$ is an irreducible $\widetilde{\operatorname{Sp}}\left(Z_{\nu}\right)$-module. Hence the map $d_{\nu} \circ r_{V_{\nu}^{\prime}, V_{\nu}}$ is surjective. It follows that for a given $\phi_{\nu} \in \mathcal{S}\left(V_{\nu}^{n}\right)$, there is $\phi_{\nu}^{\prime} \in \mathcal{S}\left(\left(V_{\nu}^{\prime}\right)^{n}\right)$ such that $f^{\phi_{\nu}}=f^{r_{V_{\nu}^{\prime}}, V_{\nu}}\left(\phi_{\nu}^{\prime}\right)$, and hence

$$
l_{\nu}\left(\omega_{\psi_{\nu}}\left(\tilde{g_{\nu}}, 1\right) \phi_{\nu}\right)=l_{\nu}\left(\omega_{\psi_{\nu}}\left(\tilde{g_{\nu}}, 1\right) r_{V_{\nu}^{\prime}, V_{\nu}}\left(\phi_{\nu}^{\prime}\right)\right),
$$

for all $\tilde{g_{\nu}} \in \operatorname{Sp}\left(Z_{\nu}\right)$. Therefore, for a given factorizable $\phi=\bigotimes_{\nu} \phi_{\nu} \in \mathcal{S}\left(V_{\mathbb{A}}^{n}\right)$, there is a factorizable $\phi^{\prime}=\bigotimes_{\nu} \phi_{\nu}^{\prime} \in \mathcal{S}\left(\left(V_{\AA}^{\prime}\right)^{n}\right)$, such that

$$
I_{\mathrm{reg}, V, \psi}(\tilde{g}, \phi)=I_{\mathrm{reg}, V, \psi}\left(\tilde{g}, r_{V^{\prime}, V}\left(\phi^{\prime}\right)\right),
$$

for all $\tilde{g} \in \operatorname{Sp} \tilde{(} Z)_{\mathbb{A}}$. Now, the identity (2.31) follows from (2.30).

Next, we are going to establish a regularized Siegel-Weil formula characterizing residues of Siegel Eisenstein series on the $k$-split even orthogonal group $\mathrm{O}(l, l)$. This was done by Moeglin for totally real number fields by using the regularization at real archimedean local place [Moe97a, § 3.2]. We use the regularization in terms of elements in a $p$-adic Hecke algebra as in [Ioh01], which extends the results of Moeglin to all number fields. This result will be used in the next section. We give the details very briefly. Once we introduce the regularization, the rest follows exactly as in [Moe97a, $\S 3.2]$.

Let $(U, b)$ be a non-degenerate quadratic space over $k$, which is $k$-split and even dimensional. Put $\operatorname{dim}_{k} U=2 l$. In this case, the double cover $\widetilde{\operatorname{Sp}}(U \otimes Z)_{\mathbb{A}}$ splits over $\operatorname{Sp}(Z)_{\mathbb{A}} \times \mathrm{O}(U)_{\mathbb{A}}$. Denote, again, by $\omega_{\psi}$ or $\omega_{\psi, Z \otimes U}$ the Weil representation of $\operatorname{Sp}(Z)_{\mathbb{A}} \times \mathrm{O}(U)_{\mathbb{A}}$ obtained by composing the $\psi$-Weil representation of $\widetilde{\operatorname{Sp}}(U \otimes Z)_{\mathbb{A}}$ with an embedding

$$
\operatorname{Sp}(Z)_{\mathbb{A}} \times \mathrm{O}(U)_{\mathbb{A}} \rightarrow \widetilde{\operatorname{Sp}}(U \otimes Z)_{\mathbb{A}}
$$

Consider the corresponding theta series $\theta_{\psi}^{\phi}(g, h)$, where $\phi$ is in a corresponding Schrödinger model $\mathcal{S}\left(U_{\mathbb{A}}^{n}\right)$. The theta integral involved in the regularized Siegel-Weil formula characterizing residues of Siegel Eisenstein series on $\mathrm{O}(U)_{\mathbb{A}}$ is given by

$$
\int_{\operatorname{Sp}(Z) \backslash \operatorname{Sp}(Z)_{\mathbb{A}}} \theta_{\psi}^{\phi}(g, h) d g,
$$

which is divergent in general if $n<l$, so that regularization is necessary.

First, we fix a local finite place $\nu_{0}$, such that $\psi_{\nu_{0}}$ is unramified and the local quadratic form $b_{\nu_{0}}$ is $k_{\nu_{0}}$-unimodular. Assume that $\phi$ is fixed by $\omega_{\psi_{\nu_{0}}}\left(K_{\nu_{0}}^{Z} \times K_{\nu_{0}}^{U}\right)$, where $K_{\nu_{0}}^{Z}$ (respectively, $K_{\nu_{0}}^{U}$ ) is a (standard) maximal compact subgroup of $\operatorname{Sp}\left(Z_{\nu_{0}}\right)$ (respectively, $\mathrm{O}\left(U_{\nu_{0}}\right)$ ). (Thus, the choice of $\nu_{0}$ depends on $\phi$.) The local Howe duality asserts that $\omega_{\psi_{\nu_{0}}}\left(\mathcal{H}_{\mathrm{O}\left(U_{\nu_{0}}\right)}\right)$ and $\omega_{\psi_{\nu_{0}}}\left(\mathcal{H}_{\mathrm{Sp}\left(Z_{\nu_{0}}\right)}\right)$ coincide as algebras of operators on $\mathcal{S}\left(U_{\nu_{0}}^{n}\right)^{\omega_{\nu_{0}}}\left(K_{\nu_{0}}^{Z} \times K_{\nu_{0}}^{U}\right)$, where $\mathcal{H}_{\mathrm{Sp}\left(Z_{\nu_{0}}\right)}$ (respectively, $\left.\mathcal{H}_{\mathrm{O}\left(U_{\nu_{0}}\right)}\right)$ is the spherical Hecke algebra of $\operatorname{Sp}\left(Z_{\nu_{0}}\right)$ (respectively, $\mathrm{O}\left(U_{\nu_{0}}\right)$ ) with respect to $K_{\nu_{0}}^{Z}$ (respectively, $K_{\nu_{0}}^{U}$ ); see [How79] and [MVW87]. Following the explicit calculation of the local theta correspondence (Howe duality) for unramified representations by Rallis in [Ral82], one has the following.

Proposition 2.3. Assume that $n \leqslant l$. Let $\theta=\theta_{l, n, \nu_{0}}$ be the Hecke algebra homomorphism

$$
\mathbb{C}\left[q^{ \pm s_{1}}, \ldots, q^{ \pm s_{l}}\right]^{W_{\mathrm{O}(U)}} \cong \mathcal{H}_{\mathrm{O}\left(U_{\nu_{0}}\right)} \stackrel{\theta}{\rightarrow} \mathcal{H}_{\mathrm{Sp}\left(Z_{\nu_{0}}\right)} \cong \mathbb{C}\left[q^{ \pm t_{1}}, \ldots, q^{ \pm t_{n}}\right]^{W_{\mathrm{Sp}}(Z)},
$$

which is given by

$$
\theta\left(q^{s_{i}}\right)= \begin{cases}q^{t_{i}}, & \text { if } 1 \leqslant i \leqslant n, \\ q^{-l+i}, & \text { if } n<i \leqslant l .\end{cases}
$$




\section{JIANG AND D. SOUDRY}

Then for all $\alpha \in \mathcal{H}_{\mathrm{O}\left(U_{\nu_{0}}\right)}$,

$$
\omega_{\psi_{\nu_{0}}, Z_{\nu_{0}}, b_{\nu_{0}}}(1, \alpha)=\omega_{\psi_{\nu_{0}}, Z_{\nu_{0}}, b_{\nu_{0}}}(\theta(\alpha), 1)
$$

as endomorphisms of $\mathcal{S}\left(U_{\nu_{0}}^{n}\right)^{\omega_{\psi_{0}}}\left(K_{\nu_{0}}^{Z} \times K_{\nu_{0}}^{U}\right)$.

We have

$$
\mathbb{C}\left[q^{ \pm s_{1}}, \ldots, q^{ \pm s_{l}}\right]^{W_{\mathrm{O}(U)}} \cong \mathbb{C}\left[X_{1}, \ldots, X_{l}\right]^{S_{l}},
$$

where $X_{i}=q^{s_{i}}+q^{-s_{i}}, i=1, \ldots, l ; S_{l}$ is the symmetric group on $l$ letters. Similarly, for $Y_{i}=q^{t_{i}}+q^{-t_{i}}$, $i=1, \ldots, n$,

$$
\mathbb{C}\left[q^{ \pm t_{1}}, \ldots, q^{ \pm t_{n}}\right]^{W_{\mathrm{Sp}(Z)}} \cong \mathbb{C}\left[Y_{1}, \ldots, Y_{n}\right]^{S_{n}}
$$

Let $\sigma_{1}, \ldots, \sigma_{l}$ denote the elementary symmetric polynomials in $X_{1}, \ldots, X_{l}$. We have, as in [KR94, Corollary 5.1.2] and [Ioh01, Lemma 1.3] that, for $n<l$, there is a unique element $\alpha_{l, n, \nu_{0}} \in \mathcal{H}_{\mathrm{O}\left(U_{\nu_{0}}\right)}$, of the form

$$
\alpha_{l, n, \nu_{0}}=\sigma_{n+1}-\sum_{i=1}^{n} a_{i} \sigma_{i}
$$

such that

$$
\theta_{l, n, \nu_{0}}\left(\alpha_{l, n, \nu_{0}}\right)=0
$$

Define, for $n \leqslant l$,

$$
\alpha_{\nu_{0}}=\alpha_{l, n-1, \nu_{0}} .
$$

This defines an element of $\mathcal{H}_{\mathrm{O}\left(U_{\nu_{0}}\right)}$. We have $\theta_{l, n-1, \nu_{0}}\left(\alpha_{\nu_{0}}\right)=0$, which means that

$$
\alpha_{\nu_{0}}\left(Y_{1}, \ldots, Y_{n-1}, q^{-l+n}+q^{l-n}, q^{-l+n+1}+q^{l-n-1}, \ldots, q^{-1}+q, 2\right)=0 .
$$

Note that $\alpha_{\nu_{0}}$ is of degree $n$ and is $S_{l}$-invariant. Consider the element $\theta\left(\alpha_{\nu_{0}}\right)=\theta_{l, n, \nu_{0}}\left(\alpha_{\nu_{0}}\right)$. Then

$$
\theta\left(\alpha_{\nu_{0}}\right)\left(Y_{1}, \ldots, Y_{n}\right)=\alpha_{\nu_{0}}\left(Y_{1}, \ldots, Y_{n}, q^{-l+n+1}+q^{l-n-1}, \ldots, q^{-1}+q, 2\right) .
$$

This is a polynomial of degree $n$ in $\mathbb{C}\left[Y_{1}, \ldots, Y_{n}\right]^{S_{n}}=\mathcal{H}_{\mathrm{Sp}\left(Z_{\nu_{0}}\right)}$. Since it is symmetric and satisfies $\theta\left(\alpha_{\nu_{0}}\right)\left(Y_{1}, \ldots, Y_{n-1}, q^{-l+n}+q^{l-n}\right)=0$, we conclude, as in [KR94, Lemma 5.5.4] and as in [Ioh01, (1.1)] that

$$
\theta\left(\alpha_{\nu_{0}}\right)\left(Y_{1}, \ldots, Y_{n}\right)=\prod_{i=1}^{n}\left(Y_{i}-\left(q^{-l+n}+q^{l-n}\right)\right) .
$$

Let $\theta\left(\alpha_{\nu_{0}}\right)$ act on the trivial representation of $\operatorname{Sp}\left(Z_{\nu_{0}}\right)$ by the scalar $c_{\alpha_{\nu_{0}}}$. Then

$$
c_{\alpha_{\nu_{0}}}=\theta\left(\alpha_{\nu_{0}}\right)\left(q^{-n}+q^{n}, q^{-n+1}+q^{n-1}, \ldots, q^{-1}+q\right) .
$$

We conclude that, for $2 n<l, c_{\alpha_{\nu_{0}}} \neq 0$.

Let us return to the global set-up. Recall that we choose the finite place $\alpha_{\nu_{0}}$, dependent on the function $\phi \in \mathcal{S}\left(U_{\mathbb{A}}^{n}\right)$. Assume that $n \leqslant l$, so that $\alpha_{\nu_{0}}=\alpha_{l, n-1, \nu_{0}}$ is defined. Then, as in [KR94, Proposition 5.3.1] and [Ioh01, Proposition 1.5], we have that $\theta_{\psi}^{\omega_{\psi_{0}}}\left(1, \alpha_{\nu_{0}}\right) \phi(g, h)$ is rapidly decreasing in $g \in \operatorname{Sp}(Z) \backslash \operatorname{Sp}\left(Z_{\mathbb{A}}\right)$, for all $h \in \mathrm{O}\left(U_{\mathbb{A}}\right)$. For this, it is enough to take $g$ as a Siegel domain and $h \in \prod_{\nu \neq \nu_{0}} \mathrm{O}\left(U_{\nu}\right)$ (since $\mathrm{O}(U) \prod_{\nu \neq \nu_{0}} \mathrm{O}\left(U_{\nu}\right)$ is dense in $\mathrm{O}\left(U_{\mathbb{A}}\right)$ ). Then, it is enough to show that $\omega_{\psi_{\nu_{0}}}\left(1, \alpha_{\nu_{0}}\right) \phi_{\nu_{0}}\left(y_{1}, \ldots, y_{n}\right)=0$, for linearly dependent $y_{1}, \ldots, y_{n} \in U_{\nu_{0}}$. We assume, for simplicity, that $\phi$ is decomposable. As in [Ioh01, p. 210] we can find $a \in \mathrm{GL}_{n}\left(\mathcal{O}_{\nu_{0}}\right)$ and $x_{1}, \ldots, x_{n-1} \in U_{\nu_{0}}$ such that $\left(y_{1}, \ldots, y_{n}\right)=\left(x_{1}, \ldots, x_{n-1}, 0\right) a$, and then, since $\phi_{\nu_{0}}$ is fixed by $K_{\nu_{0}}^{Z} \times 1$ and the action of $\alpha_{\nu_{0}}$ (via $\omega_{\psi_{\nu_{0}}}$ ) commutes with the action of (the Siegel parabolic subgroup of) $\operatorname{Sp}\left(Z_{\nu_{0}}\right)$, we get that

$$
\omega_{\psi_{\nu_{0}}}\left(1, \alpha_{\nu_{0}}\right) \phi_{\nu_{0}}\left(y_{1}, \ldots, y_{n}\right)=\omega_{\psi_{\nu_{0}}}\left(1, \alpha_{\nu_{0}}\right) \phi_{\nu_{0}}\left(x_{1}, \ldots, x_{n-1}, 0\right) .
$$


ON THE GENERICITY of CUSPIDAL AUTOMORPhic FORMS OF $\mathrm{SO}(2 n+1)$, II

The last expression is zero, since $\theta_{l, n-1, \nu_{0}}\left(\alpha_{\nu_{0}}\right)=0$. Indeed, let

$$
\left\{z_{1}, \ldots, z_{n}, z_{-n}, \ldots, z_{-1}\right\},
$$

be a symplectic basis of $Z$, as in property (d) of $\S 2.1$. Put

$$
Z^{\prime}=\operatorname{Span}_{k}\left\{z_{1}, \ldots, z_{n-1}, z_{-n+1}, \ldots, z_{-1}\right\} .
$$

Denote by $\omega_{\psi}^{\prime}$ the Weil representation for the dual pair $\operatorname{Sp}\left(Z^{\prime}\right) \times \mathrm{O}(U)$, and let us realize $\omega_{\psi}^{\prime}$ in the Schrödinger model $\mathcal{S}\left(U_{\mathbb{A}}^{n-1}\right)$. Denote the restriction of $\phi_{\nu_{0}}$ to $U_{\nu_{0}}^{n-1}$ by $\phi_{\nu_{0}}^{\prime}$. Then

$$
\begin{aligned}
\omega_{\psi_{\nu_{0}}}\left(1, \alpha_{\nu_{0}}\right) \phi_{\nu_{0}}\left(x_{1}, \ldots, x_{n-1}, 0\right) & =\omega_{\psi_{\nu_{0}}}^{\prime}\left(1, \alpha_{\nu_{0}}\right) \phi_{\nu_{0}}^{\prime}\left(x_{1}, \ldots, x_{n-1}\right) \\
& =\omega_{\psi_{\nu_{0}}}^{\prime}\left(\theta_{l, n-1, \nu_{0}}\left(\alpha_{\nu_{0}}\right), 1\right) \phi_{\nu_{0}}^{\prime}\left(x_{1}, \ldots, x_{n-1}\right) \\
& =0 .
\end{aligned}
$$

This proves that

$$
\theta_{\psi}^{\omega_{\psi_{0}}\left(1, \alpha_{\nu_{0}}\right) \phi}(g, h)=\sum_{x \in U^{n}, \operatorname{rank}(x)=n} \omega_{\psi}(g, h) \omega_{\psi_{\nu_{0}}}\left(1, \alpha_{\nu_{0}} \phi(x)\right),
$$

where, for $x=\left(x_{1}, \ldots, x_{n}\right), \operatorname{rank}(x)$ is the dimension of the subspace of $U$, spanned by $x_{1}, \ldots, x_{n}$. Now, the rapid decrease in $g$ follows as in [KR94, Proposition 5.3.1]. Moreover, if

$$
\int_{\operatorname{Sp}(Z) \backslash \operatorname{Sp}\left(Z_{\mathbb{A}}\right)} \theta_{\psi}^{\phi}(g, h) d g
$$

converges absolutely, then

$$
\int_{\operatorname{Sp}(Z) \backslash \operatorname{Sp}(Z)_{\mathbb{A}}} \theta_{\psi}^{\omega_{\psi_{\nu_{0}}}\left(1, \alpha_{\nu_{0}}\right) \phi}(g, h) d g=c_{\alpha_{\nu_{0}}} \int_{\operatorname{Sp}(Z) \backslash \operatorname{Sp}(Z)_{\mathbb{A}}} \theta_{\psi}^{\phi}(g, h) d g .
$$

As before, we define, for $2 n<l$ (so that $c_{\alpha_{\nu_{0}}} \neq 0$ )

$$
I_{\text {reg, } \psi}(h, \phi)=c_{\alpha_{\nu_{0}}}^{-1} \int_{\operatorname{Sp}(Z) \backslash \operatorname{Sp}(Z)_{\mathbb{A}}} \theta_{\psi}^{\omega_{\psi_{\nu_{0}}}\left(1, \alpha_{\nu_{0}}\right) \phi}(g, h) d g .
$$

This definition is independent of the choice of the place $\nu_{0}$.

Next, consider a Siegel parabolic subgroup $P \subset \mathrm{O}(U)$, whose Levi part is isomorphic to $\mathrm{GL}_{l}$. Take a standard section $\xi_{s}$ in the space of the normalized induced module $\operatorname{Ind}_{P_{\mathbb{A}}}^{\mathrm{O}(U)_{\mathbb{A}}}\left(|\operatorname{det}|^{s}\right)$ and form an Eisenstein series $E\left(h, \xi_{s}\right)$ as usual. By [KR90b, Theorem 1.0.1], the Eisenstein series $E\left(h, \xi_{s}\right)$ converges absolutely when the real part of $s$ is greater than $(l-1) / 2$; it has a meromorphic continuation to the complex plane $\mathbb{C}$ and, after normalization, it has at most simple poles occurring only at $s=(l-1) / 2-j \neq 0$ with $j \in\{0,1, \ldots, l-1\}$.

Assume now that $n<(l-1) / 2$. Then $s=(l-1) / 2$ is a simple pole of the normalized Eisenstein series, and it is also a pole of $E\left(h, \xi_{s}\right)$. Now, we can repeat the proof of Moeglin, in [Moe97a, $\S 3.2$ ], to get the following version of the regularized Siegel-Weil formula, which is valid for arbitrary number fields.

Theorem 2.4. Assume that $n<(l-1) / 2$. Then, for every Schwartz function $\phi$ as above, there is a section $\xi_{s}$ as above, such that

$$
\int_{\operatorname{Sp}(Z) \backslash \operatorname{Sp}(Z)_{\mathbb{A}}} \theta_{\psi}^{\omega_{\psi_{\nu_{0}}}\left(1, \alpha_{\nu_{0}}\right) \phi}(g, h) d g=\operatorname{Res}_{s=(l-1) / 2-n} E\left(h, \xi_{s}\right) .
$$




\section{JIANG AND D. SOUDRY}

\section{The theta correspondence}

We are going to prove Theorems 1.1, 1.2, and 1.3 as stated in the introduction. The main task is to give an explicit description of various spaces of automorphic functions via the theta correspondences. In $\S 3.1$, we start with an irreducible genuine cuspidal automorphic representation $\tilde{\pi}$ of $\widetilde{\operatorname{Sp}}(Z)_{\mathbb{A}}$, and in $\S 3.3$, we start with an irreducible cuspidal automorphic representation $\sigma$ of $\mathrm{O}(V)_{\mathbb{A}}$. Theorem 1.1 is proven in $\S 3.2$ and the proofs of Theorems 1.2 and 1.3 are discussed in $\S 3.3$.

\subsection{Certain subspace of automorphic forms on $\widetilde{\operatorname{Sp}}(Z)_{\mathbb{A}}$}

Let $\tilde{\pi}$ be an irreducible genuine cuspidal automorphic representation of $\widetilde{\operatorname{Sp}}(Z)_{\mathbb{A}}$. The $\psi^{-1}$-theta lift of $\tilde{\pi}$ to $\mathrm{O}(V)_{\mathbb{A}}$ is denoted by $\theta_{\psi^{-1}, Z}^{V}(\tilde{\pi})$ as before. Assume that $\theta_{\psi^{-1}, Z}^{V}(\tilde{\pi})$ is a cuspidal representation of $\mathrm{O}(V)_{\mathbb{A}}$. For any integer $a \geqslant 0$, define a symplectic vector space $Z_{a}$ by

$$
Z_{a}=Z \oplus l_{a},
$$

where $l_{a}$ denotes the $2 a$-dimensional symplectic space over $k$. We consider the $\psi$-theta lift $\theta_{\psi, V}^{Z_{a}}\left(\theta_{\psi^{-1}, Z}^{V}(\tilde{\pi})\right)$, which is a subspace of automorphic functions on the metaplectic group $\widetilde{\operatorname{Sp}}\left(Z_{a}\right)_{\mathbb{A}}$.

To fix a Rao normalized cocycle for $\widetilde{\operatorname{Sp}}\left(Z_{a}\right)_{\mathbb{A}}$ (at each place), we fix a symplectic $k$-basis

$$
B_{a}^{\prime}=\left\{z_{1}^{\prime}, \ldots, z_{a}^{\prime}, z_{-a}^{\prime}, \ldots, z_{-1}^{\prime}\right\}
$$

of $l_{a}$, and a symplectic $k$-basis

$$
B_{Z}=\left\{z_{1}, \ldots, z_{n}, z_{-n}, \ldots, z_{-1}\right\}
$$

of $Z$. Then we take the symplectic $k$-basis

$$
B_{a}=\left\{z_{1}^{\prime}, \ldots, z_{a}^{\prime}, z_{1}, \ldots, z_{n}, z_{-n}, \ldots, z_{-1}, z_{-a}^{\prime}, \ldots, z_{-1}^{\prime}\right\}
$$

for $Z_{a}$. Put

$$
l_{a}^{ \pm}=\operatorname{Span}_{F}\left\{z_{ \pm 1}^{\prime}, \ldots, z_{ \pm a}^{\prime}\right\} .
$$

Denote by $P_{a}$ the standard Siegel parabolic subgroup of $\operatorname{Sp}\left(Z_{a} \oplus Z\right)$, with respect to the basis $i_{1}\left(B_{a}\right) \cup i_{2}\left(B_{Z}\right)$, ordered as in property (b) of $\S 2.1$, where $i_{1}$ (respectively, $i_{2}$ ) is the embedding of $Z_{a}$ (respectively, $Z$ ) in the first (respectively, second) coordinate of $Z_{a} \oplus Z$. Consider, also, the homomorphism as in part (e) of $\S 2.2$ (which we now shorten to $\tilde{j}$, instead of $\tilde{j}_{\mathbb{A}}$ ),

$$
\tilde{j}: \widetilde{\operatorname{Sp}}\left(Z_{a}\right)_{\mathbb{A}} \times \widetilde{\operatorname{Sp}}(Z)_{\mathbb{A}} \rightarrow \widetilde{\operatorname{Sp}}\left(Z_{a} \times Z\right)_{\mathbb{A}} .
$$

Let $c$ be the $k$-linear automorphism of $Z$, such that $z_{i} c=z_{i}$ and $z_{-i} c=-z_{-i}$ for all $1 \leqslant i \leqslant n$. Clearly, $c \in \operatorname{GSp}(Z)$, and has similitude factor -1 . Let $c$ act by conjugation on $\operatorname{Sp}\left(Z_{\nu}\right)$, i.e.

$$
g \mapsto g^{c}=c g c^{-1} .
$$

Then one can lift this conjugation to an automorphism of $\widetilde{\operatorname{Sp}}\left(Z_{\nu}\right)$ by

$$
(g, \epsilon)=\tilde{g} \mapsto \tilde{g}^{\prime}=(g, \epsilon)^{\prime}=\left(g^{c}, \epsilon\left(x(g),(-1)^{u(g)+1}\right)(-1,-1)^{\frac{1}{2} u(g)(u(g)-1)}\right),
$$

in the notation of (2.5). See [Szp06] for details. This gives an automorphism of $\widetilde{\operatorname{Sp}}(Z)_{\mathbb{A}}, \tilde{g} \mapsto \tilde{g}^{\prime}$, which lifts the conjugation $c$ on $\operatorname{Sp}(Z)_{\mathbb{A}}$. Finally, we denote by $K_{Z}$ the standard (with respect to $B_{Z}$ ) maximal compact subgroup of $\operatorname{Sp}(Z)_{\mathbb{A}}$, and we let $\tilde{K}_{Z}$ denote its inverse image in $\widetilde{\operatorname{Sp}}(Z)_{\mathbb{A}}$. We use similar notation for other symplectic spaces.

Proposition 3.1. Let $\tilde{\pi}$ be an irreducible genuine cuspidal automorphic representation of $\widetilde{\operatorname{Sp}}(Z)_{\mathbb{A}}$ with $\operatorname{dim}_{k} Z=2 n$. Assume that $\theta_{\psi^{-1}, Z}^{V}(\tilde{\pi})$ is cuspidal on $\mathrm{O}(V)_{\mathbb{A}}$ with $\operatorname{dim}_{k} V=m$. Let $a \geqslant 1$ be an integer such that $m<n+a+1$. Then the subspace $\theta_{\psi, V}^{Z_{a}}\left(\theta_{\psi^{-1}, Z}^{V}(\tilde{\pi})\right)_{0}$ consisting of all $\tilde{K}_{Z_{a}}$-finite 
ON THE GENERICITY OF CUSPIDAL AUTOMORPHIC FORMS OF $\mathrm{SO}(2 n+1)$, II

functions of the space $\theta_{\psi, V}^{Z_{a}}\left(\theta_{\psi^{-1}, Z}^{V}(\tilde{\pi})\right)$ is contained in the subspace of automorphic forms on $\widetilde{\operatorname{Sp}}\left(Z_{a}\right)_{\mathbb{A}}$ generated by the automorphic functions

$$
\tilde{g}_{a} \mapsto \int_{C_{2} \operatorname{Sp}(Z) \backslash \widetilde{\operatorname{Sp}}(Z)_{\mathbb{A}}} \varphi_{\tilde{\pi}}(\tilde{g}) \operatorname{Res}_{s=(n+a+1-m) / 2} E\left(\tilde{j}\left(\tilde{g}_{a}, \tilde{g}^{\prime}\right), f_{s}\right) d \tilde{g},
$$

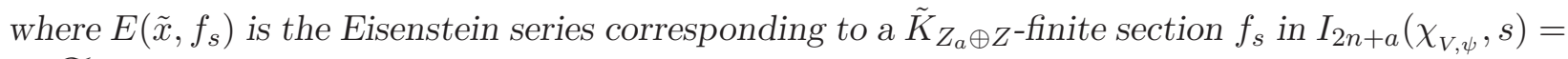
$\operatorname{Ind}_{\tilde{P}_{a}}^{\widetilde{S p}}\left(Z_{a} \oplus Z\right)_{\mathbb{A}}\left(\chi_{V, \psi}|\operatorname{det}|^{s}\right)$.

Proof. We repeat [Moe97a, $\S 2.1]$. By definition, the space $\theta_{\psi, V}^{Z_{a}}\left(\theta_{\psi^{-1}, Z}^{V}(\tilde{\pi})\right)$ is spanned by

$$
\int_{\mathrm{O}(V) \backslash \mathrm{O}(V)_{\mathbb{A}}} \theta_{\psi}^{\phi^{\prime}}\left(\tilde{g}_{a}, h\right) \int_{C_{2} \operatorname{Sp}(Z) \backslash \widetilde{\operatorname{Sp}}(Z)_{\mathbb{A}}} \theta_{\psi^{-1}}^{\phi}(\tilde{g}, h) \varphi_{\tilde{\pi}}(\tilde{g}) d \tilde{g} d h
$$

where $\varphi_{\tilde{\pi}}$ is a cusp form in the space of $\tilde{\pi}, \phi \in \mathcal{S}\left(V_{\mathbb{A}}^{n}\right), \phi^{\prime} \in \mathcal{S}\left(V_{\mathbb{A}}^{n+a}\right)$ and $\tilde{g}_{a} \in \widetilde{\operatorname{Sp}}\left(Z_{a}\right)_{\mathbb{A}}$. We have (see [MVW87, p. 36]) that for $\tilde{g} \in \widetilde{\operatorname{Sp}}(Z)_{\mathbb{A}}$,

$$
\theta_{\psi^{-1}}^{\phi}(\tilde{g}, h)=\theta_{\psi}^{\phi}\left(\tilde{g}^{\prime}, h\right)
$$

Viewing $\phi^{\prime} \otimes \phi$ as an element of $\mathcal{S}\left(V_{\mathbb{A}}^{2 n+a}\right)$, we have (see [MVW87, p. 37]) that

$$
\theta_{\psi}^{\phi^{\prime}}\left(\tilde{g}_{a}, h\right) \theta_{\psi}^{\phi}\left(\tilde{g}^{\prime}, h\right)=\theta^{\phi^{\prime} \otimes \phi}\left(\tilde{j}_{j}\left(\tilde{g}_{a}, \tilde{g}^{\prime}\right), h\right) .
$$

Hence, the integral (3.2) becomes

$$
\int_{\mathrm{O}(V) \backslash \mathrm{O}(V)_{\mathbb{A}}} \int_{C_{2} \operatorname{Sp}(Z) \backslash \widetilde{\mathrm{Sp}}(Z)_{\mathbb{A}}} \theta_{\psi}^{\phi^{\prime} \otimes \phi}\left(\tilde{j}\left(\tilde{g}_{a}, \tilde{g}^{\prime}\right), h\right) \varphi_{\tilde{\pi}}(\tilde{g}) d \tilde{g} d h .
$$

In order to interchange the order of the integrations with respect to $\tilde{g}$ and $h$, we have to regularize the integration with respect to $h$. To this end, we choose a finite place $\nu_{0}$, which satisfies all of the requirements as given in $\S 2.3$. Take an element $\alpha_{\nu_{0}}$ in the spherical Hecke algebra of $\widetilde{\operatorname{Sp}}\left(\left(Z_{a} \oplus Z\right)_{\nu_{0}}\right)$ as in $\S 2.3$. Then $\theta_{\psi}^{\omega_{\psi_{0}}\left(\alpha_{\nu_{0}}, 1\right)\left(\phi^{\prime} \otimes \phi\right)}\left(\tilde{j}\left(\tilde{g}_{a}, \tilde{g}^{\prime}\right), h\right)$ is rapidly decreasing in $h$, where $\omega_{\psi_{\nu_{0}}}$ denotes, for brevity, the Weil representation of

$$
\widetilde{\mathrm{Sp}}\left(\left(Z_{a} \oplus Z\right)_{\nu_{0}}\right) \times \mathrm{O}(V)_{\nu_{0}}
$$

Hence, we can interchange the order of integrations in (3.3) and obtain, by using (2.25), that (3.3) equals

$$
c_{\nu_{0}}^{-1} \int_{C_{2} \operatorname{Sp}(Z) \backslash \widetilde{\mathrm{Sp}}(Z)_{\mathbb{A}}} \int_{\mathrm{O}(V) \backslash \mathrm{O}(V)_{\mathbb{A}}} \theta_{\psi}^{\omega_{\psi_{\nu_{0}}}\left(\alpha_{\nu_{0}}, 1\right)\left(\phi^{\prime} \otimes \phi\right)}\left(\tilde{j}\left(\tilde{g}_{a}, \tilde{g}^{\prime}\right), h\right) d h \varphi_{\tilde{\pi}}(\tilde{g}) d \tilde{g}
$$

which can be written as

$$
\int_{C_{2} \operatorname{Sp}(Z) \backslash \widetilde{\operatorname{Sp}}(Z)_{\mathbb{A}}} I_{\mathrm{reg}, V, \psi}\left(\tilde{j}\left(\tilde{g}_{a}, \tilde{g}^{\prime}\right), \phi^{\prime} \otimes \phi\right) \varphi_{\tilde{\pi}}(\tilde{g}) d \tilde{g} .
$$

Finally the proposition follows from Corollary 2.2 .

In order to further describe the structure of the subspace $\theta_{\psi, V}^{Z_{a}}\left(\theta_{\psi^{-1}, Z}^{V}(\tilde{\pi})\right)$ as in Proposition 3.1, we have to investigate the following integral

$$
\int_{C_{2} \operatorname{Sp}(Z) \backslash \widetilde{\operatorname{Sp}}(Z)_{\mathbb{A}}} \varphi_{\tilde{\pi}}(\tilde{g}) E\left(\tilde{j}\left(\tilde{g}_{a}, \tilde{g}^{\prime}\right), f_{s}\right) d \tilde{g} .
$$

Consider the polarization

$$
Z_{a} \oplus Z=Z_{a, \Delta}^{+}+Z_{a, \Delta}^{-}
$$




\section{JIANG AND D. SOUDRY}

where $Z_{a, \Delta}^{ \pm}=i_{1}\left(l_{a}^{ \pm}\right)+\{(v, \pm v c) \mid v \in Z\}$. Note that for $g \in \operatorname{Sp}(Z), j\left(g, g^{c}\right)$ acts as identity on $i_{1}\left(l_{a}^{ \pm}\right)$, and $(v, \pm v c) j\left(g, g^{c}\right)=(v g, \pm(v g) c)$. Here, we use notation as in properties (b) and (e) of $\S 2.1$. Let $P_{a, \Delta}$ be the Siegel parabolic subgroup of $\operatorname{Sp}\left(Z_{a} \oplus Z\right)$, which preserves the maximal totally isotropic subspace $Z_{a, \Delta}^{-}$. Write its Levi decomposition $P_{a, \Delta}=M_{a, \Delta} U_{a, \Delta}$, where $M_{a, \Delta}$ is the Levi part, which is isomorphic to $\mathrm{GL}(2 n+a)$. Thus, $\beta(g)=j\left(g, g^{c}\right)$ defines an embedding of $\operatorname{Sp}(Z)$ into $M_{a, \Delta}$. Let $\delta_{0} \in \operatorname{Sp}\left(Z_{a} \oplus Z\right)$ be such that it acts as the identity on $i_{1}\left(l_{a}\right)$ and $\delta_{0} P_{a, \Delta} \delta_{0}^{-1}=P_{a}$, the standard Siegel parabolic subgroup of $\operatorname{Sp}\left(Z_{a} \oplus Z\right)$ defined before Proposition 3.1. For a standard section $f_{s}$ in $I_{2 n+a}\left(\chi_{V, \psi}, s\right)$, we define $f_{s}^{\prime}(\tilde{b})=f_{s}\left(\left(\delta_{0}, 1\right) \tilde{b}\right)$. Then

$$
f_{s}^{\prime} \in I_{2 n+a}^{\prime}\left(\chi_{V, \psi}^{\prime}, s\right)=\operatorname{Ind}_{\tilde{P}_{a, \Delta}(\mathbb{A})}^{\widetilde{\mathrm{A}_{2}}\left(Z_{a} \oplus Z\right)_{\mathbb{A}}}\left(\chi_{V, \psi}^{\prime} \cdot\left|\operatorname{det}_{Z_{a, \Delta}^{-}}\right|^{S}\right),
$$

where $\chi_{V, \psi}^{\prime}(\tilde{p})=\chi_{V, \psi}\left(\left(\delta_{0}, 1\right) \tilde{p}\left(\delta_{0}, 1\right)^{-1}\right)$. We have

$$
E\left(\tilde{x}, f_{s}\right)=E\left(\left(\delta_{0}, 1\right) \tilde{x}, f_{s}\right)=E^{\prime}\left(\tilde{x}, f_{s}^{\prime}\right),
$$

where $E^{\prime}$ denotes the Eisenstein series corresponding to $I_{2 n+a}^{\prime}\left(\chi_{V, \psi}^{\prime}, s\right)$. It is easy to check that at each place $\nu$, we have, for $p_{\nu} \in P_{a, \Delta}\left(k_{\nu}\right)$,

$$
\left(\delta_{0}, 1\right)\left(p_{\nu}, \epsilon\right)\left(\delta_{0}, 1\right)^{-1}=\left(\delta_{0} p_{\nu} \delta_{0}^{-1}, \epsilon_{\nu} c_{\nu}\left(\delta_{0}, p_{\nu}\right)\left(\operatorname{det}_{Z_{a, \Delta}^{-}}\left(p_{\nu}\right), x_{\nu}\left(\delta_{0}\right)\right)_{\nu}\right)
$$

where $c_{\nu}$ is the Rao normalized cocycle on $\widetilde{\operatorname{Sp}}\left(\left(Z_{a} \times Z\right)_{\nu}\right)$ with respect to the basis above. Note the following properties of $f_{s}^{\prime}$.

Lemma 3.2. Let $R_{a} \subset \operatorname{Sp}\left(Z_{a}\right)$ be the parabolic subgroup which preserves $l_{a}^{-}$, and its Levi decomposition $R_{a}=M_{R_{a}} U_{R_{a}}$. We identify $M_{R_{a}}$ with $\mathrm{GL}\left(l_{a}^{+}\right) \times \operatorname{Sp}(Z)$. In the following, $\epsilon= \pm 1$ and $\tilde{b} \in \widetilde{\operatorname{Sp}}\left(Z_{a}\right)_{\mathbb{A}}$.

(1) Let $u \in U_{R_{a}}(\mathbb{A})$. Then

$$
f_{s}^{\prime}\left(\tilde{j}_{1}(u, \epsilon) \tilde{b}\right)=\epsilon f_{s}^{\prime}(\tilde{b})
$$

(2) Let $d \in M_{R_{a}}(\mathbb{A})$ correspond to an element of $G L\left(l_{a}^{+}\right)_{\mathbb{A}}$. Then

$$
f_{s}^{\prime}\left(\tilde{j}_{1}(d, \epsilon) \tilde{b}\right)=\epsilon \cdot \chi_{V, \psi}\left(\operatorname{det}_{l_{a}^{+}} d\right)\left|\operatorname{det}_{l_{a}^{+}} d\right|^{s+n+(a+1) / 2} f_{s}^{\prime}(\tilde{b}) .
$$

(3) Let $g \in M_{R_{a}}(\mathbb{A})$ correspond to an element in $\operatorname{Sp}(Z)_{\mathbb{A}}$ and let $\tilde{g}$ be an inverse image of $g$ in $\widetilde{\operatorname{Sp}}(Z)_{\mathbb{A}}$. Then

$$
f_{s}^{\prime}\left(\tilde{j}_{1}(\tilde{g}) \tilde{b}\right)=f_{s}^{\prime}\left(\tilde{j}_{2}\left(\tilde{g}^{\prime}\right)^{-1} \tilde{b}\right) .
$$

Proof. By (3.7) and the fact that $j_{1}\left(U_{R_{a}}\right) \subset U_{a, \Delta}$, we have

$$
f_{s}^{\prime}\left(\tilde{j}_{1}(u, \epsilon) \tilde{b}\right)=\epsilon \cdot c\left(\delta_{0}, j_{1}(u)\right) f_{s}^{\prime}(\tilde{b}) .
$$

It is clear that $u \mapsto \tilde{j}_{1}(u, 1)=\left(j_{1}(u), 1\right)$ is an embedding of $U_{R_{a}}(\mathbb{A})$ inside $\widetilde{\operatorname{Sp}}\left(Z_{a} \times Z\right)_{\mathbb{A}}$. This implies that $u \mapsto c\left(\delta_{0}, j_{1}(u)\right)$ is a $\mu_{2}$-valued character of $U_{R_{a}}(\mathbb{A})$, and hence is trivial. This proves part (1).

For part $(2)$, since $\delta_{0}$ is the identity on $j_{1}\left(l_{a}\right)$, it follows that $\delta_{0}$ and $j_{1}(d)$ commute. Hence $\left(\delta_{0}, 1\right)$ and $\tilde{j}_{1}(d, \epsilon)$ commute. It follows that

$$
\chi_{V, \psi}^{\prime}\left(\tilde{j}_{1}(d, \epsilon)\right)=\chi_{V, \psi}\left(\tilde{j}_{1}(d, \epsilon)\right)=\epsilon \cdot \chi_{V, \psi}\left(\operatorname{det}_{l_{a}^{+}} d\right) .
$$

Now the assertion follows.

Finally, we have $f_{s}^{\prime}\left(\tilde{j}_{1}(\tilde{g}) \tilde{b}\right)=f_{s}^{\prime}\left(\tilde{j}\left(\tilde{g}, \tilde{g}^{\prime}\right) \tilde{j}_{2}\left(\tilde{g}^{\prime}\right)^{-1} \tilde{b}\right)$. Note that $j\left(g, g^{c}\right)$ lies in $M_{R_{a}}(\mathbb{A})$ and its determinant on either of $Z_{a, \Delta}^{ \pm}(\mathbb{A})$ is $\operatorname{det} g=1$. It follows that $f_{s}^{\prime}\left(\tilde{j}_{1}(\tilde{g}) \tilde{b}\right)=\chi_{V, \psi}^{\prime}\left(\tilde{j}\left(\tilde{g}, \tilde{g}^{\prime}\right)\right) f_{s}^{\prime}\left(\tilde{j}_{2}\left(\tilde{g}^{\prime}\right)^{-1} \tilde{b}\right)$. Clearly, $\tilde{g} \mapsto \chi_{V, \psi}^{\prime}\left(\tilde{j}\left(\tilde{g}, \tilde{g}^{\prime}\right)\right)$ is a $\mu_{2}$-valued character of $\widetilde{\operatorname{Sp}}(Z)_{\mathbb{A}}$, trivial on $C_{2}(\mathbb{A})$, and hence, it is trivial. This proves part (3). 
ON THE GENERICITY OF CUSPIDAL AUTOMORPHIC FORMS OF $\mathrm{SO}(2 n+1)$, II

Motivated by the global integral (3.5), we define, as in [Moe97a, p. 211],

$$
f_{\varphi_{\tilde{\pi}, s}}\left(\tilde{g}_{a}\right)=\int_{C_{2} \backslash \widetilde{\operatorname{Sp}}(Z)_{\mathbb{A}}} \varphi_{\tilde{\pi}}(\tilde{g}) f_{s}^{\prime}\left(\tilde{j}\left(\tilde{g}_{a}, \tilde{g}^{\prime}\right)\right) d \tilde{g}
$$

As in [Moe97a, $\S 2.1]$, the previous lemma implies that, if the integrals (3.8) converge absolutely at a point $s$, then $f_{\varphi_{\tilde{\pi}, s}}$ is a $\tilde{K}_{Z_{a}}$-finite element of the representation

$$
J(\tilde{\pi}, s)=\operatorname{Ind}_{\tilde{R}_{a}(\mathbb{A})}^{\widetilde{S p}\left(Z_{a}\right)_{\mathbb{A}}}\left(\mu_{\psi}\left|\operatorname{det}_{l_{a}^{+}}\right|^{s} \otimes \tilde{\pi}\right),
$$

where $\mu_{\psi}\left|\operatorname{det}_{l_{a}^{+}}\right|^{s} \otimes \tilde{\pi}$ is the representation of $\tilde{R}_{a}(\mathbb{A})$, which is trivial on $\left(U_{R_{a}}(\mathbb{A}), 1\right)$ and acts as

$$
\epsilon \cdot \chi_{V, \psi}\left(\operatorname{det}_{l_{a}^{+}} d\right)\left|\operatorname{det}_{l_{a}^{+}} d\right|^{s} \tilde{\pi}(\tilde{g})
$$

for an element $(d, \epsilon) \tilde{g}$ in the Levi part $M_{R_{a}}(\mathbb{A})$ with $d \in \operatorname{GL}\left(l_{a}^{+}\right)_{\mathbb{A}}$ and $\tilde{g} \in \widetilde{\operatorname{Sp}}(Z)_{\mathbb{A}}$. Note that $\left|\operatorname{det}_{l_{a}^{+}} d\right|^{n+(a+1) / 2}=\delta_{R_{a}}^{\frac{1}{2}}(d)$.

Proposition 3.3. The integral (3.8) converges absolutely for $\operatorname{Re}(s)>n+(a+1) / 2$, and continues to a meromorphic function in the whole plane. Its poles are contained in the set of poles of an Eisenstein series corresponding to a certain fixed (independent of $\tilde{\pi}$ ) degenerate principal series for $\widetilde{\mathrm{Sp}}(Z \oplus Z)_{\mathbb{A}}$, induced from a Siegel parabolic subgroup.

Proof. As in [Moe97a, $\S 2,(2)]$, let us rewrite

$$
\begin{aligned}
f_{\varphi_{\tilde{\pi}, s}}\left(\tilde{g}_{a}\right) & =\int_{C_{2} \backslash \widetilde{\mathrm{Sp}}(Z)_{\mathbb{A}}} \varphi_{\tilde{\pi}}(\tilde{g}) f_{s}^{\prime}\left(\tilde{j}_{2}\left(\tilde{g}^{\prime}\right) \tilde{j}_{1}\left(\tilde{g}_{a}\right)\right) d \tilde{g} \\
& =\int_{C_{2} \operatorname{Sp}(Z) \backslash \widetilde{\operatorname{Sp}}(Z)_{\mathbb{A}}} \varphi_{\tilde{\pi}}(\tilde{g}) \sum_{\gamma \in \operatorname{Sp}(Z)} f_{s}^{\prime}\left(\left(j_{2}(\gamma), 1\right) \tilde{j}_{2}\left(\tilde{g}^{\prime}\right) \tilde{j}_{1}\left(\tilde{g}_{a}\right)\right) d \tilde{g}
\end{aligned}
$$

Note that $(\gamma, 1)^{\prime}=\left(\gamma^{c}, 1\right)$, for $\gamma \in \operatorname{Sp}(Z)$. Since $\gamma \mapsto j_{2}(\gamma)$ is an embedding of $\operatorname{Sp}(Z)$ inside $P_{a, \Delta} \backslash \operatorname{Sp}\left(Z_{a} \times Z\right)$, the inner sum in the integral (3.10) is a sub-series of the series defining the Eisenstein series $E^{\prime}\left(\tilde{j}\left(\tilde{g}_{a}, \tilde{g}^{\prime}\right), f_{s}^{\prime}\right)$ as in $(3.4)$, and hence it converges absolutely for $\operatorname{Re}(s)>n+$ $(a+1) / 2$.

In order to obtain the analytic continuation, we may assume that $\tilde{g}_{a}=\tilde{h}$ lies in $\widetilde{\mathrm{Sp}}(Z)_{\mathbb{A}} \subset$ $\tilde{M}_{R_{a}}(\mathbb{A})$. Then we saw that $\tilde{h} \mapsto f_{\varphi_{\tilde{\pi}, s}}(\tilde{h})$ lies in the space of $\tilde{\pi}$. Thus, it is enough to show the analytic continuation of

$$
\int_{C_{2} \operatorname{Sp}(Z) \backslash \widetilde{\operatorname{Sp}}(Z)_{\mathbb{A}}} f_{\varphi_{\tilde{\pi}, s}}(\tilde{h}) \bar{\xi}_{\tilde{\pi}}(\tilde{h}) d \tilde{h},
$$

for every cusp form $\xi_{\tilde{\pi}}$. By using part (3) of Lemma 3.2, the integral (3.11), in its convergence domain, is equal to

$$
\begin{aligned}
& \int_{C_{2} \operatorname{Sp}(Z) \backslash \widetilde{\operatorname{Sp}}(Z)_{\mathbb{A}}} \int_{C_{2} \backslash \widetilde{\operatorname{Sp}}(Z)_{\mathbb{A}}} \varphi_{\tilde{\pi}}(\tilde{g}) \bar{\xi}_{\tilde{\pi}}(\tilde{h}) f_{s}^{\prime}\left(\tilde{j}_{1}(\tilde{h}) \tilde{j}_{2}\left(\tilde{g}^{\prime}\right)\right) d \tilde{g} d \tilde{h} \\
& =\int_{C_{2} \operatorname{Sp}(Z) \backslash \widetilde{\operatorname{Sp}}(Z)_{\mathbb{A}}} \int_{C_{2} \backslash \widetilde{\operatorname{Sp}}(Z)_{\mathbb{A}}} \varphi_{\tilde{\pi}}(\tilde{g}) \bar{\xi}_{\tilde{\pi}}(\tilde{h}) f_{s}^{\prime}\left(\tilde{j}_{2}\left(\tilde{h}^{-1} \tilde{g}^{\prime}\right)\right) d \tilde{g} d \tilde{h} \\
& =\int_{C_{2} \operatorname{Sp}(Z) \backslash \widetilde{\operatorname{Sp}}(Z)_{\mathbb{A}}} \int_{C_{2} \backslash \widetilde{\operatorname{Sp}}(Z)_{\mathbb{A}}} \varphi_{\tilde{\pi}}(\tilde{h} \tilde{g}) \bar{\xi}_{\tilde{\pi}}(\tilde{h}) f_{s}^{\prime}\left(\tilde{j}_{2}\left(\tilde{g}^{\prime}\right)\right) d \tilde{g} d \tilde{h} \\
& =\int_{C_{2} \backslash \widetilde{\operatorname{Sp}}(Z)_{\mathbb{A}}} f_{s}^{\prime}\left(\tilde{j}_{2}\left(\tilde{g}^{\prime}\right)\right)\left\langle\tilde{\pi}(\tilde{g}) \varphi_{\tilde{\pi}}, \xi_{\tilde{\pi}}\right\rangle_{L^{2}} d \tilde{g},
\end{aligned}
$$




\section{JIANG AND D. SOUDRY}

where $\langle,\rangle_{L^{2}}$ is the standard $L^{2}$-product of cuspidal automorphic forms on $C_{2} \operatorname{Sp}(Z) \backslash \widetilde{\operatorname{Sp}}(Z)_{\mathbb{A}}$. Note that the switch of order of integrations $d \tilde{g}$ and $d \tilde{h}$ is justified, since $\varphi_{\tilde{\pi}}$ and $\xi_{\tilde{\pi}}$ are rapidly decreasing. Thus, the integral (3.11), using (3.8), is equal to

$$
\int_{C_{2} \backslash \widetilde{\mathrm{Sp}}(Z)_{\mathbb{A}}} f_{\mathcal{S}}^{\prime}\left(\tilde{j}_{1}(\tilde{g})\right)\left\langle\varphi_{\tilde{\pi}}, \tilde{\pi}(\tilde{g}) \xi_{\tilde{\pi}}\right\rangle_{L^{2}} d \tilde{g} .
$$

We recognize (3.12) as the result of unfolding the Rankin-Selberg integrals of the doubling method applied to metaplectic groups. This unfolding is quite formal, and is obtained exactly as in the linear case. See [PR86] and [LR05]. Thus, let $f_{s}^{*}$ be the restriction of $f_{s}^{\prime}$ to $\widetilde{\operatorname{Sp}}(Z \oplus Z)_{\mathbb{A}}$. Then $f_{s}^{*}$ is a holomorphic $\tilde{K}_{Z \times Z}$-finite section in

$$
\operatorname{Ind}_{\tilde{P}}^{\widetilde{\operatorname{Sp}}(Z \oplus Z)_{\mathbb{A}}}\left(\chi_{V, \psi}^{\prime}|\operatorname{det}|^{s+a / 2}\right) .
$$

Here $P_{\Delta}=P_{0, \Delta}$, and we think of $\operatorname{Sp}(Z \oplus Z)$ as $\operatorname{Sp}\left(Z_{0} \oplus Z\right)$. Let $E^{*}\left(\tilde{h}, f_{s}^{*}\right)$ be the corresponding Eisenstein series on $\widetilde{\operatorname{Sp}}(Z \oplus Z)_{\mathbb{A}}$. Then (3.12) equals

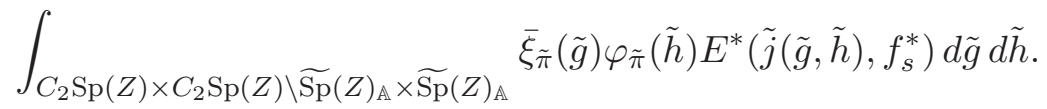

This integral is meromorphic in the whole plane, and its poles are included in the set of poles of $E^{*}\left(\tilde{x}, f_{s}^{*}\right)$.

Remark 3.4. Note that the integral (3.12) is Eulerian, and its computation at unramified places was carried out by $\mathrm{Li}$ in [Li92]. Also, it is easy to see that integral (3.5) is (for $a=0$ ) an inner integral of (3.13).

Let $E^{R_{a}}\left(\tilde{h}, f_{\varphi_{\tilde{\pi}, s}}\right)$ denote the Eisenstein series on $\widetilde{\operatorname{Sp}}\left(Z_{a}\right)_{\mathbb{A}}$ corresponding to the section $f_{\varphi_{\tilde{\pi}, s}}$ given by (3.8). This Eisenstein series will give another description of the subspace $\theta_{\psi, V}^{Z_{a}}\left(\theta_{\psi^{-1}, Z}^{V}(\tilde{\pi})\right)$.

THEOREM 3.5. In the notation above, we have

$$
\int_{C_{2} \operatorname{Sp}(Z) \backslash \widetilde{\operatorname{Sp}}(Z)_{\mathbb{A}}} \varphi_{\tilde{\pi}}(\tilde{g}) E^{\prime}\left(\tilde{j}\left(\tilde{g}_{a}, \tilde{g}^{\prime}\right), f_{s}^{\prime}\right) d \tilde{g}=E^{R_{a}}\left(\tilde{g}, f_{\varphi_{\tilde{\pi}, s}}\right) .
$$

Proof. The proof is exactly as in [Moe97a, §2], which is a generalization of the doubling method. The reason for the exact similarity is the fact that $\operatorname{Sp}(Z)$ (respectively, $\operatorname{Sp}\left(Z_{a} \oplus Z\right)$ ) is a subgroup of $\widetilde{\operatorname{Sp}}(Z)_{\mathbb{A}}$ (respectively, $\widetilde{\operatorname{Sp}}\left(Z_{a} \oplus Z\right)_{\mathbb{A}}$ ), and similarly for unipotent radicals (over $\mathbb{A}$ ). Thus, when we unwind the left-hand side of (3.14), we have to examine the double coset space

$$
P_{a, \Delta} \backslash \operatorname{Sp}\left(Z_{a} \oplus Z\right) / j\left(\operatorname{Sp}\left(Z_{a}\right) \times \operatorname{Sp}(Z)\right) .
$$

This is described in [PR86] and used in [Moe97a, $\S 2$ ], and exactly as in [Moe97a, $\S 2$ ] only one double coset has a non-trivial contribution to the left-hand side of (3.14), and it is the double coset of the identity element: $P_{a, \Delta} j\left(\operatorname{Sp}\left(Z_{a}\right) \times \operatorname{Sp}(Z)\right)$. Again, the reason is that for the other double cosets, we get a stabilizer in $\operatorname{Sp}\left(Z_{a}\right) \times \operatorname{Sp}(Z)$, whose projection on $\operatorname{Sp}(Z)$ contains a unipotent radical, which contributes an inner integration of a cusp form of $\tilde{\pi}$ along this radical, and hence results in zero. Thus, we get, as in [Moe97a, §2], that the left-hand side of (3.14) equals

$$
\sum_{\gamma \in R_{a}(k) \backslash \operatorname{Sp}\left(Z_{a}\right)} \int_{C_{2} \backslash \widetilde{\operatorname{Sp}}(Z)_{\mathbb{A}}} \varphi_{\tilde{\pi}}(\tilde{g}) f_{s}^{\prime}\left(\tilde{j}\left((\gamma, 1) \tilde{g}_{a}, \tilde{g}^{\prime}\right)\right) d \tilde{g}=\sum_{\gamma \in R_{a}(k) \backslash \operatorname{Sp}\left(Z_{a}\right)} f_{\varphi_{\tilde{\pi}}, s}\left((\gamma, 1) \tilde{g}_{a}\right)=E^{R_{a}}\left(\tilde{g}_{a}, f_{\varphi_{\tilde{\pi}}, s}\right) .
$$

This proves the theorem.

Using Proposition 3.1 and (3.6), we conclude as follows. 
THEOREM 3.6. Let $\tilde{\pi}$ be an irreducible genuine cuspidal automorphic representation of $\widetilde{\operatorname{Sp}}(Z)_{\mathbb{A}}$. Assume that $\theta_{\psi^{-1}, Z}^{V}(\tilde{\pi})$ is cuspidal. Let a be a positive integer such that $m<n+a+1$. Then the space $\theta_{\psi, V}^{Z_{a}}\left(\theta_{\psi^{-1}, Z}^{V}(\tilde{\pi})\right)$ is contained in the space of automorphic forms on $\widetilde{\operatorname{Sp}}\left(Z_{a}\right)_{\mathbb{A}}$ generated by the residual automorphic forms

$$
\tilde{g}_{a} \mapsto \operatorname{Res}_{s=(n+a+1-m) / 2} E^{R_{a}}\left(\tilde{g}_{a}, f_{\varphi_{\tilde{\pi}}, s}\right) .
$$

\subsection{Proof of Theorem 1.1}

We are now ready to complete the proof of Theorem 1.1 stated in the introduction. The details are exactly as in [Moe97a, Moe97b]. We indicate them briefly. Let us apply the constant term along $U_{R_{a}}$ (as an operator) to the inclusion asserted in Theorem 3.6. For an automorphic form $\xi$, on $\widetilde{\operatorname{Sp}}\left(Z_{a}\right)_{\mathbb{A}}$, denote by $\xi_{U_{R_{a}}}$ its constant term along $U_{R_{a}}$, restricted to the Levi part $\widetilde{M}_{R_{a}}(\mathbb{A})$. For an automorphic representation $\tau$ of $\widetilde{\operatorname{Sp}}\left(Z_{a}\right)_{\mathbb{A}}$, we denote by $\tau_{U_{R_{a}}}$ the automorphic representation of $\tilde{M}_{R_{a}}(\mathbb{A})$ whose space consists all of the $\xi_{U_{R_{a}}}$, as $\xi$ varies in the space of $\tau$. Recall that $M_{R_{a}}$ is isomorphic to $\mathrm{GL}\left(l_{a}^{+}\right) \times \mathrm{Sp}(Z)$. By Rallis' tower property [Ral84], for any cuspidal automorphic representation $\sigma$ of $\mathrm{O}(V)_{\mathbb{A}}$,

$$
\operatorname{Res}_{\widetilde{\operatorname{Sp}}(Z)_{\mathbb{A}}}\left[\theta_{\psi, V}^{Z_{a}}(\sigma)\right]_{U_{R_{a}}}=\theta_{\psi, V}^{Z}(\sigma)
$$

Here we view $\widetilde{\operatorname{Sp}}(Z)_{\mathbb{A}}$ as a subgroup of $\widetilde{M}_{R_{a}}(\mathbb{A})$. Moreover, $\widetilde{\mathrm{GL}}\left(l_{a}^{+}\right)_{\mathbb{A}}$ acts on $\left[\theta_{\psi, V}^{Z_{a}}(\sigma)\right]_{U_{R_{a}}}$ by the character

$$
(d, \epsilon) \mapsto \epsilon \chi_{V, \psi}\left(\operatorname{det}_{l_{a}^{+}} d\right)\left|\operatorname{det}_{l_{a}^{+}} d\right|^{m / 2}=\mu_{\psi}(d)\left|\operatorname{det}_{l_{a}^{+}} d\right|^{m / 2} .
$$

The main formula which explains (3.16) and (3.17) is that, for $\phi \in S\left(V_{\mathbb{A}}^{n+a}\right)$, of the form $\phi_{1} \otimes \phi_{2}$, where $\phi_{1} \in \mathcal{S}\left(V_{\mathbb{A}}^{a}\right)$ and $\phi_{2} \in \mathcal{S}\left(V_{\mathbb{A}}^{n}\right)$ (in the notation of $(2.25)$ ),

$$
\theta_{\psi}^{\phi}\left(\varphi_{\sigma}\right)_{U_{R_{a}}}((d, \epsilon) \tilde{g})=\epsilon \phi_{1}(0) \mu_{\psi}(d)|\operatorname{det}(d)|^{m / 2} \theta_{\psi}^{\phi_{2}}\left(\varphi_{\sigma}\right)(\tilde{g})
$$

for $\tilde{g} \in \widetilde{\mathrm{Sp}}(Z)_{\mathbb{A}}, d \in \mathrm{GL}\left(l_{a}^{+}\right)_{\mathbb{A}}, \epsilon= \pm 1$. From Theorem 3.6, we conclude that, over $\widetilde{M}_{R_{a}}(\mathbb{A})$,

$$
\mu_{\psi}\left|\operatorname{det}_{l_{a}^{+}}\right|^{m / 2} \otimes \theta_{\psi, V}^{Z}\left(\theta_{\psi^{-1}, Z}^{V}(\tilde{\pi})\right) \subset\left\{\left[\operatorname{Res}_{s=(n+a+1-m) / 2} E^{R_{a}}\left(\cdot, f_{\varphi_{\tilde{\pi}, s}}\right)\right]_{U_{R_{a}}} \mid \varphi_{\tilde{\pi}} \in V_{\tilde{\pi}}\right\} .
$$

Exactly as in [Moe97a, $\S 2]$, by expressing the constant term along $U_{R_{a}},\left[E^{R_{a}}\left(\cdot, f_{\varphi_{\tilde{\pi}, s}}\right)\right]_{U_{R_{a}}}$, of the Eisenstein series $E^{R_{a}}\left(\cdot, f_{\varphi_{\tilde{\pi}, s}}\right)$ in terms of intertwining operators, we can replace the right-hand side of (3.19) by a certain sum of residues of certain intertwining operators, which take values in the space of $\tilde{\pi}$. The details are exactly as in [Moe97a, $\S 2$ ], only that we replace the characters

$$
\operatorname{diag}\left(t_{1}, \ldots, t_{a}\right) \mapsto\left|t_{1}\right|^{s_{1}} \cdots\left|t_{a}\right|^{s_{a}}
$$

there by (our) characters,

$$
\left(\operatorname{diag}\left(t_{1}, \ldots, t_{a}\right), \epsilon\right) \mapsto \epsilon \chi_{V, \psi}\left(t_{1} \cdots t_{a}\right)\left|t_{1}\right|^{s_{1}} \cdots\left|t_{a}\right|^{s_{a}} .
$$

We then conclude from $(3.19)$, by considering only the action of $\widetilde{\operatorname{Sp}}(Z)_{\mathbb{A}}$, that

$$
\theta_{\psi, V}^{Z}\left(\theta_{\psi^{-1}, Z}^{V}(\tilde{\pi})\right)=\tilde{\pi}
$$

This concludes the proof of part (1) of Theorem 1.1.

The proof of part (2) of Theorem 1.1 now follows, as in [Moe97a, $\S 2$ ], the only interesting case being $b \leqslant m-n-1$. Otherwise, we just apply Theorem 3.6 for $a=b$. For a fixed integer $a>m-n-1$, we follow the same process as in the proof of part (1) of Theorem 1.1 above. This time we apply the constant term along $U_{R_{a-b}}$ (as an operator) to the inclusion asserted in Theorem 3.6, viewing $Z_{a}$ as $\left(Z_{a-b}\right)_{b}$. This will prove part (2) of Theorem 1.1 .

Finally, part (3) of Theorem 1.1 follows immediately from part (1) of Theorem 1.1 and the Rallis tower property [Ral84]. Indeed, since $\theta_{\psi^{-1}, Z}^{V}(\tilde{\pi})$ is assumed to be cuspidal, then any irreducible 


\section{JIANG AND D. SOUDRY}

sub-representation of it, $\sigma$, is cuspidal. Then $\theta_{\psi, V}^{Z}(\sigma)$ is non-trivial and is a sub-representation of $\theta_{\psi, V}^{Z}\left(\theta_{\psi^{-1}, Z}^{V}(\tilde{\pi})\right)=\tilde{\pi}$, and hence equals $\tilde{\pi}$ and, in particular, is cuspidal. By the Rallis tower

property [Ral84], $\theta_{\psi, V}^{Z_{-b}}(\sigma)=0$ for all positive integers $b$. This proves part (3) of Theorem 1.1 and hence completes the proof of the whole theorem.

\subsection{Proof of Theorems 1.2 and 1.3}

The proofs of these two theorems can be directly inferred from [Moe97b]. For this, let us explain how to proceed until we reach the point where we can directly use the results of [Moe97b].

Let $\sigma$ be an irreducible cuspidal automorphic representation of $\mathrm{O}(V)_{\mathbb{A}}$. Assume that $\theta_{\psi^{-1}, V}^{Z}(\sigma)$ is cuspidal, as an automorphic representation of $\widetilde{\operatorname{Sp}}(Z)_{\mathbb{A}}$. Let $V_{b}$ be the orthogonal direct sum of the quadratic space $V$ (equipped with its symmetric form $Q$ ) and $b$ hyperbolic planes $k u_{i}+k u_{-i}$, $i=1, \ldots, b$. As usual, $u_{i}$ and $u_{-i}$ are isotropic, and the Gram matrix of the pair $\left(u_{i}, u_{-i}\right)$ is $\left(\begin{array}{ll}0 & 1 \\ 1 & 0\end{array}\right)$.

Put $\ell_{b}=\bigoplus_{i=1}^{b}\left(k u_{i}+k u_{-i}\right)$ and $\ell_{b}^{ \pm}=\bigoplus_{i=1}^{b} k u_{ \pm i}$. Denote by $Q_{b}$ the symmetric bilinear form on $V_{b}$. Consider the theta lift $\theta_{\psi, Z}^{V_{b}}\left(\theta_{\psi^{-1}, V}^{Z}(\sigma)\right)$. Its space is generated by the following automorphic forms on $\mathrm{O}\left(V_{b}\right)_{\mathbb{A}}$

$$
\int_{C_{2} \operatorname{Sp}(Z) \backslash \widetilde{\operatorname{Sp}}(Z)_{\mathbb{A}}} \theta_{\psi}^{\phi_{2}}\left(\tilde{g}, h_{b}\right) \int_{\mathrm{O}(V) \backslash \mathrm{O}(V)_{\mathbb{A}}} \theta_{\psi^{-1}}^{\phi_{1}}(\tilde{g}, h) \varphi_{\sigma}(h) d h d \tilde{g} .
$$

Here $h_{b} \in \mathrm{O}\left(V_{b}\right)_{\mathbb{A}}, \phi_{1} \in \mathcal{S}\left(V_{\mathbb{A}}^{n}\right), \phi_{2} \in \mathcal{S}\left(V_{b}(\mathbb{A})^{n}\right)$, and $\varphi_{\sigma}$ is a cusp form in the space of $\sigma$. Now, let us view $\omega_{\psi^{-1}, Z \otimes V}$ as $\omega_{\psi, Z \otimes V^{\prime}}$, where $V^{\prime}$ denotes the space $V$, equipped with the symmetric form $-Q$. (See [MVW87, p. 36].) We have a natural embedding over $k$

$$
\mathrm{O}\left(V_{b}\right) \times \mathrm{O}\left(V^{\prime}\right) \rightarrow \mathrm{O}\left(V_{b} \oplus V^{\prime}\right)
$$

where the symmetric form of $V_{b} \oplus V^{\prime}$ is $Q_{b} \oplus(-Q)$. Note that $V_{b} \oplus V^{\prime}$ is a totally split, evendimensional quadratic space over $k$. We have [MVW87, p. 37]

$$
\theta_{\psi, Z \otimes V_{b}}^{\phi_{2}}\left(\tilde{g}, h_{b}\right) \theta_{\psi, Z \otimes V^{\prime}}^{\phi_{1}}(\tilde{g}, h)=\theta_{\psi, Z \otimes\left(V_{b} \oplus V^{\prime}\right)}^{\phi_{2} \otimes \phi_{1}}\left(g,\left(h_{b}, h\right)\right),
$$

where $g \in \operatorname{Sp}(Z)_{\mathbb{A}}$ is the projection of $\tilde{g}$, and we think of $\phi_{2} \otimes \phi_{1}$ as an element of $\mathcal{S}\left(\left(V_{b} \oplus V^{\prime}\right)_{\mathbb{A}}^{n}\right)$. Thus, (3.20) becomes

$$
\int_{\mathrm{Sp}(Z) \backslash \mathrm{Sp}(Z)_{\mathbb{A}}} \int_{\mathrm{O}\left(V^{\prime}\right) \backslash \mathrm{O}\left(V^{\prime}\right)_{\mathbb{A}}} \theta_{\psi, Z \otimes\left(V_{b} \oplus V^{\prime}\right)}^{\phi_{2} \otimes \phi_{1}}\left(g,\left(h_{b}, h\right)\right) \varphi_{\sigma}(h) d h d g .
$$

Now, we are at the situation of [Moe97a, $\S 1$ ]. Indeed, we assume that $2 n<m+b-1$. By applying Theorem 2.4 with regularization in terms of a $p$-adic Hecke element $\alpha$, which is the same as in the proof in $[\mathrm{Moe} 97 \mathrm{a}]$, the integral (3.21) has the form

$$
\int_{\mathrm{O}\left(V^{\prime}\right) \backslash \mathrm{O}\left(V^{\prime}\right)_{\mathbb{A}}} \varphi_{\sigma}(h) \operatorname{Res}_{s=(m+b-1) / 2-n} E\left(\left(h_{b}, h\right), \xi_{s}\right) d h .
$$

Here $\xi_{s}$ is a $K_{V_{b} \oplus V^{\prime}}$-finite section of $\operatorname{Ind}_{L_{b, \Delta}(\mathbb{A})}^{\mathrm{O}\left(V_{b} \oplus V^{\prime}\right)_{\mathbb{A}}}\left|\operatorname{det}_{V_{b, \Delta}}\right|^{s}$, and $E\left(\cdot, \xi_{s}\right)$ denotes the corresponding Eisenstein series. We denote by $L_{b, \Delta}$ the maximal parabolic subgroup of $\mathrm{O}\left(V_{b} \oplus V^{\prime}\right)$, which preserves the isotropic subspace

$$
V_{b, \Delta}=\bigoplus_{i=1}^{b} k\left(u_{i}, 0\right)+\left\{(v, v) \in V \times V^{\prime}\right\} .
$$

The proof proceeds from this point on, word by word, as in [Moe97a, $\S 1]$. Note that the parity of $m$ is no longer relevant to the rest of the proof. First, we conclude, as in the previous section, as follows. 
TheOREm 3.7. Let $\sigma$ be an irreducible cuspidal automorphic representation of $\mathrm{O}(V)_{\mathbb{A}}$. Assume that $\theta_{\psi^{-1}, V}^{Z}(\sigma)$ is cuspidal. Then $\theta_{\psi, Z}^{V_{b}} \theta_{\psi^{-1}, V}^{Z}(\sigma)$ is contained in the space of residues at $s=(m+b-1) / 2-n$ of the Eisenstein series corresponding to $\operatorname{Ind}_{D_{b}(\mathbb{A})}^{\mathrm{O}\left(V_{b}\right)_{\mathbb{A}}}\left|\operatorname{det}_{\ell_{b}^{+}}\right|^{s}$, where $D_{b}$ is the parabolic subgroup of $\mathrm{O}\left(V_{b}\right)$, which preserves $\ell_{b}^{+}$.

Now Theorem 1.2 in the introduction follows in exactly the same way as the proof of Theorem 1.1 in the previous section. Theorem 1.3 in the introduction follows from Theorems 1.1 and 1.2, exactly as in [Moe97a, § 2]. We omit the details.

\section{Applications}

In this section, we let $H_{n}=\mathrm{SO}_{2 n+1}=\mathrm{SO}(V)$, where $(V, Q)$ is the column space of dimension $2 n+1$ over $k$, equipped with the non-degenerate $k$-split symmetric bilinear form in $2 n+1$ variables associated to the symmetric matrix defined inductively by

$$
J_{2 n+1}=\left(\begin{array}{lll}
0 & & 1 \\
& J_{2 n-1} & \\
1 & & 0
\end{array}\right) .
$$

We denote by $\widetilde{G}_{n}\left(k_{\nu}\right)$ and $\widetilde{G}_{n}(\mathbb{A})$ the local and the global metaplectic groups, respectively, corresponding to the symplectic group $G_{n}=\mathrm{Sp}_{2 n}$ over $k$, with respect to the standard symplectic form on the $2 n$-dimensional row space. For an irreducible cuspidal automorphic representation $\sigma$ of $H_{n}(\mathbb{A})$, we denote by $\widetilde{\theta}_{\psi, n}^{m}(\sigma)_{+}$the $\psi$-theta lift of $\sigma$ to $\widetilde{G}_{m}(\mathbb{A})$; it is generated by the automorphic forms $\theta_{\psi}^{\phi}\left(\varphi_{\sigma}\right)_{+}$, which are defined as in (2.18), only that the integration in (2.18) is replaced by an integration over $H_{n}(k) \backslash H_{n}(\mathbb{A})$. Similarly, for an irreducible genuine cuspidal automorphic representation $\widetilde{\pi}$ of $\widetilde{G}_{m}(\mathbb{A})$, we denote by $\theta_{\psi, m}^{n}(\tilde{\pi})$ the $\psi$-theta lift of $\tilde{\pi}$ to $\mathrm{O}_{2 n+1}(\mathbb{A})$. We denote by $\theta_{\psi, m}^{n}(\tilde{\pi})_{+}$ the automorphic representation of $H_{n}(\mathbb{A})$ obtained by restricting the functions of $\theta_{\psi, m}^{n}(\tilde{\pi})$ to $H_{n}(\mathbb{A})$.

Our first application is the proof of Theorem 1.4 in the introduction, which is part (4) of the main theorem in [Fur95] without the assumptions made there.

TheOREm 4.1. Let $\sigma$ be an irreducible cuspidal automorphic representation of $H_{n}(\mathbb{A})$. Assume that $\sigma$ is generic, i.e. has a nonzero Whittaker Fourier coefficient. Then the $\psi$-theta lift of $\sigma$ to $\widetilde{G}_{n}(\mathbb{A})$, $\widetilde{\theta}_{\psi, n}^{n}(\sigma)_{+}$, is non-trivial if and only if $L\left(\sigma, \frac{1}{2}\right) \neq 0$, where $L(\sigma, s)$ is the standard $L$-function attached to $\sigma$.

Proof. Since $\sigma$ is cuspidal and generic, the local components of $\sigma$ are all unitary and locally generic, and hence, by the exponent structure of the generic unitary dual [LMT04], $L\left(\sigma_{\nu}, s\right)$ is holomorphic at $s=\frac{1}{2}$, for all places $\nu$. Thus, $L\left(\sigma, \frac{1}{2}\right) \neq 0$, if and only if $L^{S}\left(\sigma, \frac{1}{2}\right) \neq 0$, for any finite set $S$ of places of $k$, which includes the set of all archimedean places of $k$. This can also be deduced from the explicit Langlands functorial transfer from $\mathrm{SO}_{2 n+1}(\mathbb{A})$ to $\mathrm{GL}_{2 n}(\mathbb{A})$ for irreducible generic cuspidal automorphic representations $\sigma$ of $\mathrm{SO}_{2 n+1}(\mathbb{A})$, which has been established through [CKPS04, GRS01, JS03, JS04].

Choose $S$, such that $\sigma$ and $\psi$ are unramified outside $S$. If $L^{S}\left(\sigma, \frac{1}{2}\right) \neq 0$, then by [JS, Remark 2.3], $\sigma$ has a Bessel model of special type with respect to $\mathrm{SO}_{1,1}$. Then by [Fur95, Proposition 1] (with $\lambda=1$ ), $\tilde{\theta}_{\psi, n}^{n}(\sigma)_{+}$has a non-trivial $\psi$-Whittaker Fourier coefficient, and hence $\widetilde{\theta}_{\psi, n}^{n}(\sigma)_{+}$is nonzero. Note that, for this direction, we did not use any of the results proved in the previous sections.

Conversely, assume that $\widetilde{\theta}_{\psi, n}^{n}(\sigma)_{+} \neq 0$. Then we claim that $\widetilde{\theta}_{\psi, n}^{n}(\sigma)_{+}$is cuspidal. Indeed, if this is not so, then the Rallis tower property [Ral84] (which is clearly valid for special orthogonal groups as well) implies that, for the first integer $m>0$ such that $\widetilde{\theta}_{\psi, n}^{m}(\sigma)_{+} \neq 0$, the 


\section{JIANG AND D. SOUDRY}

representation $\tilde{\pi}^{\prime}=\widetilde{\theta}_{\psi, n}^{m}(\sigma)_{+}$is cuspidal. Choose an irreducible summand $\tilde{\pi}$ of $\tilde{\pi}^{\prime}$. In particular, at a given finite place $\nu$ of $k$, we get that $\tilde{\pi_{\nu}}$ is a local $\psi_{\nu}$-Howe lift of the generic $\sigma_{\nu}$, but this is impossible by [JS03, Proposition 2.1], since $m<n$. This proves the claim.

Let us denote by $Z_{2}=\left\{ \pm I_{2 n+1}\right\}$ the center of $\mathrm{O}_{2 n+1}$. Note that $\mathrm{O}_{2 n+1}=Z_{2} \times H_{n}$. Let $\mu$ be a character of $Z_{2} \backslash Z_{2}(\mathbb{A})$, and denote by $\sigma_{\mu}$ the extension of $\sigma$ to $\mathrm{O}_{2 n+1}(\mathbb{A})$ by the character $\mu$ on the center. This also allows us to extend cusp forms of $\sigma$ from $H_{n}(\mathbb{A})$ to $\mathrm{O}_{2 n+1}(\mathbb{A})$ in the same way. The space of $\widetilde{\theta}_{\psi, n}^{n}(\sigma)_{+}$is generated by the integrals $\theta_{\psi}^{\phi}\left(\varphi_{\sigma}\right)(\tilde{g})_{+}$. It is clear that

$$
\theta_{\psi}^{\phi}\left(\varphi_{\sigma}\right)(\tilde{g})_{+}=\sum_{\mu} \int_{\mathrm{O}_{2 n+1}(F) \backslash \mathrm{O}_{2 n+1}(\mathbb{A})} \theta_{\psi}^{\phi}(\tilde{g}, h) \varphi_{\sigma_{\mu}}(h) d h .
$$

Thus, it follows that there is $\mu$ such that $\tilde{\pi}=\widetilde{\theta}_{\psi, n}^{n}\left(\sigma_{\mu}\right) \neq 0$ and is, of course, cuspidal. By Theorem 1.2 , we know that $\tilde{\pi}$ is irreducible and $\sigma_{\mu}=\theta_{\psi^{-1}, n}^{n}(\tilde{\pi})$. By [Fur95, Proposition 3], we get that $\tilde{\pi}$ is $\psi$-generic since $\sigma$ is generic. Note that by restricting to $H_{n}(\mathbb{A})$, we get that $\sigma=\theta_{\psi^{-1}, n}^{n}(\tilde{\pi})_{+}$. By [CKPS04], $\sigma$ has a functorial lift to $\mathrm{GL}_{2 n}(\mathbb{A})$ and by [GRS01, Sou05], this lift has the form $\tau=\tau_{1} \boxplus \cdots \boxplus \tau_{l}$, which is an isobaric sum of $l$ pairwise non-equivalent, irreducible, cuspidal, automorphic representations $\tau_{i}$ of $\mathrm{GL}_{2 n_{i}}(\mathbb{A})$, with the property that $L^{S}\left(\tau_{i}, \Lambda^{2}, s\right)$ has a pole at $s=1$ for each $i$. It follows that with respect to $\psi, \tilde{\pi}$ lifts at almost all places to $\tau$, and by [Sou05, Theorem 13], we conclude also that $L^{S}\left(\tau_{i}, \frac{1}{2}\right) \neq 0$ for all $i$. This implies that $L^{S}\left(\sigma, \frac{1}{2}\right) \neq 0$.

Next, we are going to consider the cases when the central value of the standard $L$-function $L^{S}\left(\sigma, \frac{1}{2}\right)$ is zero.

Let $\sigma$ be an irreducible generic cuspidal automorphic representation of $H_{n}(\mathbb{A})$. By [HP83, Theorem 5.7], the $\psi$-theta lift of $\sigma$ to $\widetilde{G}_{n+1}(\mathbb{A}), \widetilde{\theta}_{\psi, n}^{n+1}(\sigma)_{+}$, is nonzero and admits non-trivial $\psi$-Whittaker Fourier coefficients. The calculation of this $\psi$-Whittaker Fourier coefficient can also be found in $[$ Fur95, $\S 4]$; its precise local variant appears in [JS03, Corollary 2.2]. The precise formula for the $\psi$-Whittaker Fourier coefficient of automorphic functions in $\widetilde{\theta}_{\psi, n}^{n+1}(\sigma)_{+}$is as follows.

Let $U$ be the standard maximal unipotent subgroup of $G_{n}$. Since the Rao cocycle is trivial on $U$ at all places, we may regard $U_{\mathbb{A}}$ as a subgroup of $\widetilde{G}_{n}(\mathbb{A})$. Denote by $\psi_{U}$ the standard nondegenerate (or Whittaker) character of $U_{\mathbb{A}}$ given by evaluating $\psi$ at the sum of all simple root coordinates of elements of $U_{\mathbb{A}}$. Let $\varphi_{\sigma}$ be a cusp form in the space of $\sigma$. Let $\omega_{\psi, V}^{n+1}$ denote the restriction of the $\psi$-Weil representation to the subgroup $\widetilde{G}_{n+1}(\mathbb{A}) \cdot H_{n}(\mathbb{A})$, realized in the Schrödinger model $\mathcal{S}\left(V_{\mathbb{A}}^{n+1}\right)$, where $V$ is the $(2 n+1)$-dimensional column space, over $k$, on which $H_{n}$ acts. Let $\left\{v_{1}, \ldots, v_{n}, v_{0}, v_{-n}, \ldots, v_{-1}\right\}$ be the standard basis of $V$ over $k$, i.e. the spans of the first $n$ vectors and of the last $n$ vectors are maximal totally isotropic subspaces, both orthogonal to the vector $v_{0}$, $Q\left(v_{i}, v_{-j}\right)=\delta_{i, j}$ for $i, j \leqslant n$, and $Q\left(v_{0}, v_{0}\right)=1$. For $\phi \in \mathcal{S}\left(V_{\mathbb{A}}^{n+1}\right)$, we denote, as before but with more precision, by $\widetilde{\theta}_{\psi, n}^{\phi, n+1}\left(\varphi_{\sigma}\right)_{+}$the $\psi$-theta lift of the cusp form $\varphi_{\sigma}$ in the space of $\sigma$ to $\widetilde{G}_{n+1}(\mathbb{A})$, which is defined as in (2.18), but with the integration taking place over $H_{n}(k) \backslash H_{n}(\mathbb{A})$. Consider the $\psi$-Whittaker Fourier coefficient of $\widetilde{\theta}_{\psi, n}^{\phi, n+1}\left(\varphi_{\sigma}\right)_{+}$

$$
\mathcal{W}_{\widetilde{\theta}_{\psi, n}^{\phi, n+1}\left(\varphi_{\sigma}\right)_{+}}^{\psi}(\tilde{g})=\int_{U \backslash U_{\mathbb{A}}} \widetilde{\theta}_{\psi, n}^{\phi, n+1}\left(\varphi_{\sigma}\right)_{+}(u \tilde{g}) \psi^{-1}(u) d u .
$$

Then we have the following expression

$$
\mathcal{W}_{\widetilde{\theta}_{\psi, n}^{\phi, n+1}\left(\varphi_{\sigma}\right)_{+}}^{\psi}(\tilde{g})=\int_{C_{n}(\mathbb{A}) \backslash H_{n}(\mathbb{A})} \omega_{\psi, V}^{n+1}(\tilde{g}, h) \phi\left(v_{1}, \ldots, v_{n}, v_{0}\right) \mathcal{W}_{\varphi_{\sigma}}^{\psi}(h) d h .
$$

Here $\mathcal{W}_{\varphi_{\sigma}}^{\psi}$ is the standard $\psi$-Whittaker Fourier coefficient of $\varphi_{\sigma}$, and $C_{n}$ is the pointwise stabilizer in $H_{n}$ of $v_{1}, \ldots, v_{n}, v_{0}$. Clearly, the right-hand side of (4.1) is not identically zero, since $\sigma$ is generic. 
Thus, if $L\left(\sigma, \frac{1}{2}\right)=0$, then by Theorem 4.1 , the $\psi$-theta lift to $\widetilde{G}_{n}(\mathbb{A}), \widetilde{\theta}_{\psi, n}^{n}(\sigma)_{+}$, vanishes. Then by the Rallis tower property [Ral84] the $\psi$-theta lift to $\widetilde{G}_{n+1}(\mathbb{A}), \widetilde{\theta}_{\psi, n}^{n+1}(\sigma)_{+}$is cuspidal. As we did before, there is a character $\mu$ of $Z_{2} \backslash Z_{2}(\mathbb{A})$ such that $\widetilde{\theta}_{\psi, n}^{n+1}\left(\sigma_{\mu}\right)=\tilde{\pi}$ is irreducible, cuspidal, and $\psi$-generic.

Conversely, let $\tilde{\pi}$ be an irreducible genuine cuspidal automorphic representation of $\widetilde{G}_{n+1}(\mathbb{A})$. Assume that $\tilde{\pi}$ is $\psi$-generic. If the $\psi^{-1}$-theta lift of $\tilde{\pi}$ to $\mathrm{O}_{2 n+1}(\mathbb{A}), \sigma^{\prime}=\theta_{\psi^{-1}, n+1}^{n}(\tilde{\pi})$, does not vanish, then $\sigma^{\prime}$ is cuspidal, according to [JS03, Corollary 2.2(2)]. By Theorems 1.1 and 1.3, we get that $\sigma^{\prime}$ is irreducible, and hence it is of the form $\sigma_{\mu}$ with notation as above. We conclude that $\tilde{\pi}=\tilde{\theta}_{\psi, n}^{n+1}\left(\sigma_{\mu}\right)$. Since $\tilde{\pi}$ is $\psi$-generic, then we can compute the $\psi$-Whittaker Fourier coefficient on $\tilde{\pi}$ as we did in (4.1), and obtain the same formula, except that, in the right-hand side of (4.1), we integrate over $C_{n}(\mathbb{A}) \backslash \mathrm{O}_{2 n+1}(\mathbb{A})$. In particular, $\mathcal{W}_{\varphi_{\sigma}}^{\psi}$ is not identically zero, and this means that $\theta_{\psi, n+1}^{n}(\tilde{\pi})_{+}$is generic. From Theorem 4.1, we also conclude that $L\left(\sigma, \frac{1}{2}\right)=0$. Let us summarize the above discussion as follows.

TheOREm 4.2. With notation above, the following hold.

(1) Let $\sigma$ be an irreducible generic cuspidal automorphic representation of $H_{n}(\mathbb{A})$. Assume that $L\left(\sigma, \frac{1}{2}\right)=0$. Then $\widetilde{\theta}_{\psi, n}^{n+1}(\sigma)_{+}$is cuspidal and $\psi$-generic.

(2) Let $\tilde{\pi}$ be an irreducible genuine $\psi$-generic cuspidal automorphic representation of $\widetilde{G}_{n+1}$. Assume that $\sigma=\theta_{\psi^{-1}, n+1}^{n}(\tilde{\pi})_{+}$is non-trivial. Then $\sigma$ is irreducible, cuspidal, and generic, such that $L\left(\sigma, \frac{1}{2}\right)=0$.

Finally, we are ready to complete our proof of Theorem 1.5. We are going to use the notation introduced in [JS] freely.

Let $\sigma$ be an irreducible cuspidal automorphic representation of $H_{n}(\mathbb{A})$, which has a nonzero Bessel model of special type, i.e. of type $\left(D_{\lambda}, 1, \psi_{n, n-1 ; \lambda}\right)$, with respect to the quadratic extension of $k$ generated by the square root of $\lambda \in k^{\times} \backslash\left(k^{\times}\right)^{2}$. See [JS, $\left.\S 2.2\right]$ for the definition of this notion. By [JS, Theorem 4.1(1)], we know that $\widetilde{\theta}_{\psi, n}^{k}(\sigma)_{+}=0$, for $k<n-1$, and by [Fur95, Proposition 1], the last space is non-trivial for $k=n$.

Assume now that $\widetilde{\theta}_{\psi, n}^{n-1}(\sigma)_{+} \neq 0$. Then, as before, we can find a character $\mu$ of $Z_{2} \backslash Z_{2}(\mathbb{A})$ such that $\tilde{\tau}=\widetilde{\theta}_{\psi, n}^{n-1}\left(\sigma_{\mu}\right)$ is cuspidal and irreducible. Again it follows from Theorems 1.2 and 1.3 that $\sigma_{\mu}=\theta_{\psi^{-1}, n-1}^{n}(\tilde{\tau})$, and then that $\sigma=\theta_{\psi^{-1}, n-1}^{n}(\tilde{\tau})_{+}$. Now, we can compute explicitly the Bessel model of special type of $\sigma=\theta_{\psi^{-1}, n-1}^{n}(\tilde{\tau})_{+}$. It is defined by the integral in [JS, (2.11)], taking there $r=n-1$ and the automorphic form $\phi$ to be the constant function 1. Then the calculation is exactly as in the $p$-adic case in [JS, Proof of Theorem 4.3]. We get that the Bessel model of special type (given by the integral) of $\sigma=\theta_{\psi^{-1}, n-1}^{n}(\tilde{\tau})_{+}$can be expressed in terms of the $\psi_{\lambda}$-Whittaker Fourier coefficient of $\tilde{\tau}$. Hence, $\widetilde{\tau}$ must be $\psi_{\lambda}$-generic. (This calculation shows that starting with an irreducible, automorphic, cuspidal representation $\tilde{\tau}$ of $\tilde{G}_{n-1}(\mathbb{A})$, which is $\psi_{\lambda^{-} \text {-generic, } \theta_{\psi^{-1}, n-1}^{n}(\tilde{\tau})_{+}}$ has a non-trivial Bessel model of special type, with respect to $\lambda$, as above, and, in particular, this theta-lift is nontrivial.) This proves the following theorem.

TheOREm 4.3. Let $\sigma$ be an irreducible cuspidal automorphic representation of $H_{n}(\mathbb{A})$. Assume that $\sigma$ has a nonzero Bessel model of special type, i.e. of type $\left(D_{\lambda}, 1, \psi_{n, n-1 ; \lambda}\right)$, with a nonsquare $\lambda \in k^{\times}$. If the $\psi$-theta lift to $\widetilde{G}_{n-1}(\mathbb{A}), \widetilde{\theta}_{\psi, n}^{n-1}(\sigma)_{+}$, does not vanish, then the automorphic representation $\widetilde{\theta}_{\psi, n}^{n-1}(\sigma)_{+}$of $\widetilde{G}_{n-1}(\mathbb{A})$ is cuspidal and $\psi_{\lambda}$-generic.

We briefly recall, from [JS], what we have proved for irreducible cuspidal automorphic representations $\sigma$ of $H_{n}(\mathbb{A})$, which have a nonzero Bessel model of special type, i.e. of type $\left(D_{\lambda}, 1, \psi_{n, n-1 ; \lambda}\right)$, with $\lambda \in k^{\times}$. We proved in [JS, $\left.\S 2.3\right]$ that if this special Bessel model is $k$-split, i.e. $\lambda \in\left(k^{\times}\right)^{2}$, then the automorphic representation $\sigma$ is generic [JS, Proposition 2.2]. 


\section{JIANG AND D. SOUDRY}

If the special Bessel model of type $\left(D_{\lambda}, 1, \psi_{n, n-1 ; \lambda}\right)$ is not $k$-split, i.e. $\lambda$ is not a square, then we proved in [JS, Theorem 4.1] that the first occurrence of the $\psi$-theta lift of $\sigma$ to the $\widetilde{G}_{m}(\mathbb{A})$-tower, i.e. the first index $m=m_{\psi}(\sigma)$, such that $\widetilde{\theta}_{\psi, n}^{m}(\sigma)_{+}$is nonzero, satisfies the following inequalities

$$
n-1 \leqslant m_{\psi}(\sigma) \leqslant n .
$$

Then we proved that if $m_{\psi}(\sigma)=n$, then there are two possibilities. The first is that $\sigma$ is nearly equivalent to an irreducible generic cuspidal automorphic representation $\sigma_{n}^{\prime}$ of $H_{n}(\mathbb{A})$, such that $\sigma_{n}^{\prime} \otimes \chi_{\lambda}$ is in the image of the $\psi^{-1}$-theta lift, from irreducible, genuine, automorphic, cuspidal, $\psi$-generic representations of $\widetilde{G}_{n}(\mathbb{A})$. In this case, we conclude, by Theorem 4.1 , that $L\left(\sigma^{\prime} \otimes \chi_{\lambda}, \frac{1}{2}\right)$ $\neq 0$. Here $\chi_{\lambda}$ is the composition of $\alpha_{\lambda}$ with the spinor norm, and $\alpha_{\lambda}$ is the character given by the Hilbert symbol $(\cdot, \lambda)_{k}$.

The second possibility is that $\sigma$ is a CAP representation with respect to the cuspidal data

$$
\left(P_{1} ; \alpha_{\lambda}|\cdot|^{\frac{1}{2}} \otimes \sigma_{n-1}\right),
$$

where $P_{1}$ is the standard parabolic subgroup of $H_{n}$, which preserves an isotropic line, and $\sigma_{n-1}$ is an irreducible cuspidal automorphic representation of $H_{n-1}(\mathbb{A})$, such that $\sigma_{n-1} \otimes \chi_{\lambda}$ is in the image of the $\psi^{-1}$-theta lift from an irreducible, genuine, automorphic, cuspidal, $\psi$-generic representation of $\widetilde{G}_{n}(\mathbb{A})$. Note that in [JS, Theorem 4.1(2)], we were unable to determine further explicit properties of the cuspidal data, in particular, of $\sigma_{n-1}$, so that we were unable to complete the proof of the CAP conjecture [JS, Conjecture 1.1] for this case. Now by Theorem 4.2, we conclude that $\sigma_{n-1}$ is generic and that the central value of the standard $L$-function twisted by $\chi_{\lambda}, L\left(\sigma_{n-1} \otimes \chi_{\lambda}, \frac{1}{2}\right)$, is zero. This proves a little more than what the CAP conjecture asserts in this case.

Now, let us analyze the remaining case, where the first occurrence $m_{\psi}(\sigma)$ is $n-1$. By Theorem 4.3, we conclude that the cuspidal automorphic representation $\widetilde{\theta}_{\psi, n}^{n-1}(\sigma)_{+}$is $\psi_{\lambda}$-generic. Thus, there is an irreducible $\psi_{\lambda}$-generic cuspidal automorphic representation $\tilde{\tau}$ of $\widetilde{G}_{n-1}(\mathbb{A})$ such that $\sigma=$ $\theta_{\psi^{-1}, n-1}^{n}(\tilde{\tau})_{+}$(by Theorems 1.2 and 1.3). In this case, we proved in [JS, Theorem 4.6] that either $\sigma$ is CAP with respect to the cuspidal data

$$
\left(P_{1} ;|\cdot|^{\frac{1}{2}} \otimes \sigma_{n-1}\right),
$$

or $\sigma$ is $\mathrm{CAP}$ with respect to the cuspidal data

$$
\left(P_{1,1} ;|\cdot| \frac{1}{2} \otimes \alpha_{\lambda}|\cdot|^{\frac{1}{2}} \otimes \sigma_{n-2}\right) .
$$

In the first case $\sigma_{n-1}$ is cuspidal and generic on $H_{n-1}(\mathbb{A})$. The proof there shows, in this case, that $\sigma_{n-1} \otimes \chi_{\lambda}$ is the image under the $\psi^{-1}$-theta lift of the $\psi$-generic representation $\widetilde{\tau}^{\lambda}$, which is an outer conjugation of $\tilde{\tau}$ by the similitude element $\operatorname{diag}\left(I_{n}, \lambda I_{n}\right)$. By Theorem 4.1, we get that the central value of the standard $L$-function twisted by $\chi_{\lambda}, L\left(\sigma_{n-1} \otimes \chi_{\lambda}, \frac{1}{2}\right)$ is nonzero.

In the second case, $P_{1,1}$ is the standard parabolic subgroup whose Levi part is isomorphic to $G L_{1} \times G L_{1} \times H_{n-2}$, and $\sigma_{n-2}$ is an irreducible cuspidal automorphic representation of $H_{n-2}(\mathbb{A})$. By construction,

$$
\sigma_{n-2} \otimes \chi_{\lambda}=\widetilde{\theta}_{\psi, n-1}^{n-2}\left(\tilde{\tau}^{\lambda}\right)_{+}
$$

Again, in [JS] we were unable to prove further properties for $\sigma_{n-2}$, so that the CAP conjecture can be completely verified for this case. Now, by Theorem $4.2, \sigma_{n-2}$ is also generic and the central value of the standard $L$-function twisted by $\chi_{\lambda}, L\left(\sigma_{n-2} \otimes \chi_{\lambda}, \frac{1}{2}\right)$ must be zero.

The proof of Theorem 1.5 is finally completed. 
ON THE GENERICITY of CUSPIDAL AUTOMORPhic FORMS OF $\mathrm{SO}(2 n+1)$, II

\section{ACKNowledgements}

Some of the work related to this paper was carried out during our participation to the special program at ESI, 2006. We would like to thank the organizers S. Kudla, M. Rapoprot and J. Schwermer for their warm invitation and thank ESI for the productive research environment and hospitality. Finally, we would like to thank the referee for very helpful comments.

\section{REFERENCES}

CKPS04 J. Cogdell, H. Kim, I. Piatetski-Shapiro and F. Shahidi, Functoriality for classical groups, Publ. Math. Inst. Hautes Études Sci. 99 (2004), 163-233.

Fur95 M. Furusawa, On the theta lift from $\mathrm{SO}_{2 n+1}$ to $\widetilde{\mathrm{Sp}}_{n}$, J. reine angew. Math. 466 (1995), 87-110.

GRS01 D. Ginzburg, S. Rallis and D. Soudry, Generic automorphic forms on $\mathrm{SO}(2 n+1)$ : functorial lift to $\mathrm{GL}(2 n)$, endoscopy, and base change, Internat. Math. Res. Notices 14 (2001), 729-764.

HP83 H. Howe and I. Piatetski-Shapiro, Some examples of automorphic forms on $\mathrm{Sp}_{4}$, Duke Math. J. 50 (1983), 55-106.

How79 R. Howe, $\theta$-series and invariant theory, in Automorphic forms, representations, and L-functions, Proceedings of Symposia in Pure Mathematics Vol. 33, Part I (American Mathematical Society, Providence, RI, 1979), 275-285.

Ioh01 A. Ichino, On a regularized Siegel-Weil formula, J. reine angew. Math. 539 (2001), 201-234.

JS03 D. Jiang and D. Soudry, The local converse theorem for $\mathrm{SO}(2 n+1)$ and applications, Ann. of Math. (2) 157 (2003), 743-806.

JS04 D. Jiang and D. Soudry, Generic representations and local Langlands reciprocity law for p-adic $\mathrm{SO}_{2 n+1}$, in Contributions to Automorphic Forms, Geometry and Number Theory, A volume in honor of Joseph S. Shalika, eds H. Hida, D. Ramakrishnan and F. Shahidi (The Johns Hopkins University Press, Baltimore, MD, 2004), 457-519.

JS D. Jiang and D. Soudry, On the genericity of cuspidal automorphic forms on $\mathrm{SO}_{2 n+1}$, J. reine angew. Math., to appear.

Kud94 S. Kudla, Splitting metaplectic covers of dual reductive pairs, Israel J. Math. 87 (1994), 361-401.

Kud96 S. Kudla, Notes on the local theta correspondence, in Lecture series in the European School in Group Theory, Schloss Hirschberg, September 1996, http://www.math.umd.edu/ ssk/ssk.research.html.

KR90a S. Kudla and S. Rallis, Degenerate principal series and invariant distributions, Israel J. Math. 69 (1990), 25-45.

KR90b S. Kudla and S. Rallis, Poles of Eisenstein series and L-functions, Israel Math. Conf. Proc. 3 (1990), 81-110.

KR94 S. Kudla and S. Rallis, A regularized Siegel-Weil formula: the first term identity, Ann. of Math. (2) 140 (1994), 1-80.

LMT04 E. Lapid, G. Muić and M. Tadić, On the generic unitary dual of quasisplit classical groups, Internat. Math. Res. Notices 26 (2004), 1335-1354.

LR05 E. Lapid and S. Rallis, On the local factors of representations classical groups, in Automorphic representations, L-functions and applications: Progress and Prospects. Proc. conf. honoring Steve Rallis, Ohio State University Mathematical Research Institute Publications, vol. 11, eds J. Cogdell, D. Jiang, S. Kudla, D. Soudry and R. Stanton (de Gruyter, Berlin, 2005), 309-360.

Li92 J.-S. Li, Non-vanishing theorems for the cohomology of certain arithmetic quotients, J. reine angew. Math. 428 (1992), 177-217.

Moe97a C. Moeglin, Non nullité de certains relêvements par séries théta, J. Lie Theory 7 (1997), 201-229.

Moe97b C. Moeglin, Quelques propriétés de base des séries théta, J. Lie Theory 7 (1997), 231-238.

MVW87 C. Moeglin, M.-F. Vignéras and J.-L. Waldspurger, Correspondances de Howe sur un corps p-adique, Lecture Notes in Mathematics, vol. 1291 (Springer, Berlin, 1987). 
ON THE GENERICITY OF CUSPIDAL AUTOMORPHIC FORMS OF $\mathrm{SO}(2 n+1)$, II

PR86 I. Piatetski-Shapiro and S. Rallis, $\epsilon$ factors for representations of classical groups, Proc. Natl. Acad. Sci. USA 83 (1986), 4589-4593.

Ral82 S. Rallis, Langlands functoriality and the Weil representation, Amer. J. Math. 104 (1982), 469-517.

Ral84 S. Rallis, On the Howe duality conjecture, Compositio Math. 51 (1984), 333-399.

Ran93 R. Ranga Rao, On some explicit formulas in the theory of Weil representation, Pacific J. Math. 157 (1993), 335-371.

Sou05 D. Soudry, On Langlands functoriality from classical groups to $\mathrm{GL}_{n}$, in Formes Automorphes (I). Actes du semestre du CEB, printemps 2000, eds J. Tilouine, H. Carayol, M. Harris and M.-F. Vigneras, Astérisque 298 (2005), 335-390.

Szp06 D. Szpruch, Uniqueness of Whittaker model for the metaplectic group, Preprint (2006).

Zhu07 S. T. Lee and C.-B. Zhu, Degenerate principal series and local theta correspondence III: The case of complex groups, Preprint (2007).

Dihua Jiang dhjiang@math.umn.edn

School of Mathematics, University of Minnesota, 206 Church Street SE, Minneapolis, MN 55455, USA

David Soudry soundry@post.tau.ac.il

School of Mathematical Sciences, Tel Aviv University, Tel Aviv, 69978, Israel 